\title{
HAUSDORFF DIMENSION OF DIVERGENT DIAGONAL GEODESICS ON PRODUCT OF FINITE VOLUME HYPERBOLIC SPACES
}

\author{
LEI YANG*
}

\begin{abstract}
In this article, we consider the product space of several non-compact finite volume hyperbolic spaces, $V_{1}, V_{2}, \ldots, V_{k}$ of dimension $n$. Let $\mathrm{T}^{1}\left(V_{i}\right)$ denote the unit tangent bundle of $V_{i}$ for each $i=1, \ldots, k$, then for every $\left(v_{1}, \ldots, v_{k}\right) \in \mathrm{T}^{1}\left(V_{1}\right) \times \cdots \times \mathrm{T}^{1}\left(V_{k}\right)$, the diagonal geodesic flow $g_{t}$ is defined by $g_{t}\left(v_{1}, \ldots, v_{k}\right)=$ $\left(g_{t} v_{1}, \ldots, g_{t} v_{k}\right)$. And we define$$
\mathfrak{D}_{k}=\left\{\left(v_{1}, \ldots, v_{k}\right) \in \mathrm{T}^{1}\left(V_{1}\right) \times \cdots \times \mathrm{T}^{1}\left(V_{k}\right): g_{t}\left(v_{1}, \ldots, v_{k}\right) \text { divergent, as } t \rightarrow \infty\right\} .
$$

We will prove that the Hausdorff dimension of $\mathfrak{D}_{k}$ is equal to $k(2 n-1)-\frac{n-1}{2}$. This extends the result of Yitwah Cheung 2].

\section{INTRODUCTION}

In 2], Yitwah Cheung considers the following interesting problem.

Let $\mathcal{M}_{k}$ be the product space of $k$ copies of $\mathrm{SL}(2, \mathbb{Z}) \backslash \mathrm{SL}(2, \mathbb{R})$,

$$
\mathcal{M}_{k}:=(\mathrm{SL}(2, \mathbb{Z}) \backslash \mathrm{SL}(2, \mathbb{R}))^{k}
$$

Let $A$ denote the diagonal subgroup of $\mathrm{SL}(2, \mathbb{R})$ :

$$
A:=\left\{a(t)=\left[\begin{array}{ll}
e^{t} & \\
& e^{-t}
\end{array}\right]: t \in \mathbb{R}\right\} .
$$

The diagonal action of $A$ on $\mathcal{M}_{k}$ is defined as follows: for $\left(v_{1}, v_{2}, \ldots, v_{k}\right) \in \mathcal{M}_{k}$, where $v_{i} \in \operatorname{SL}(2, \mathbb{Z}) \backslash \operatorname{SL}(2, \mathbb{R})$ for $i=1,2, \ldots, k$,

$$
\left(v_{1}, v_{2}, \ldots, v_{k}\right) a(t):=\left(v_{1} a(t), v_{2} a(t), \ldots, v_{k} a(t)\right) \in \mathcal{M}_{k} .
$$

The divergent set $\mathcal{D}_{k}$ is defined to be collection of points $\left(v_{1}, v_{2}, \ldots, v_{k}\right) \in \mathcal{M}_{k}$ such that $a(t)\left(v_{1}, v_{2}, \ldots, v_{k}\right)$ diverges as $t \rightarrow+\infty$. One could ask what the Hausdorff dimension of $\mathcal{D}_{k}$ is.

From geometric point of view, $\mathrm{SL}(2, \mathbb{Z}) \backslash \mathrm{SL}(2, \mathbb{R})$ can be identified as the unit tangent bundle of the hyperbolic space $\operatorname{SL}(2, \mathbb{Z}) \backslash \mathbb{H}^{2}$. The action of $A=\{a(t): t \in \mathbb{R}\}$ is the geodesic flow $\left\{g_{t}: t \in \mathbb{R}\right\}$ on $\mathrm{T}^{1}\left(\mathrm{SL}(2, \mathbb{Z}) \backslash \mathbb{H}^{2}\right)$. So $\mathcal{M}_{k}$ can be regarded as product of $k$ copies of unit tangent bundle of hyperbolic space $\mathrm{SL}(2, \mathbb{Z}) \backslash \mathbb{H}^{2}$, and the diagonal action of $A^{+}$is the diagonal geodesic flow $g_{t} \times g_{t} \times \cdots \times g_{t}$ on $\mathcal{M}_{k}$. $\mathcal{D}_{k}$ is regarded as

$$
\mathcal{D}_{k}:=\left\{\left(v_{1}, v_{2}, \ldots, v_{k}\right) \in \mathcal{M}_{k}:\left(g_{t} v_{1}, g_{t} v_{2}, \ldots, g_{t} v_{k}\right) \rightarrow \infty \text { as } t \rightarrow+\infty\right\} .
$$

When $k=1$, the problem is easy. According to a result of Dani $[\underline{6}$, for $v \in \mathrm{SL}(2, \mathbb{Z}) \backslash \mathrm{SL}(2, \mathbb{R}), a(t) v$ diverges as $t \rightarrow+\infty$ if and only if $v$ belongs to some closed $U$-orbit in $\operatorname{SL}(2, \mathbb{Z}) \backslash \operatorname{SL}(2, \mathbb{R})$, where

$$
U:=\left\{u(x)=\left[\begin{array}{ll}
1 & x \\
0 & 1
\end{array}\right]: x \in \mathbb{R}\right\}
$$

denotes the horocyclic subgroup contracted by $A^{+}:=\{a(t): t>0\}$. Therefore the Hausdorff dimension is equal to $\operatorname{dim} \mathcal{M}_{1}-1=2$.

When $k \geq 2$, the problem becomes interesting and complicated. It turns out that most divergent trajectories have non-divergent projection to each component, that is to say, most $\left(v_{1}, v_{2}, \ldots, v_{k}\right) \in \mathcal{D}_{k}$ satisfies that for each $i=1,2, \ldots, k,\left\{a(t) v_{i}: t>0\right\}$ does not diverge as $t \rightarrow+\infty$. In [2], Cheung showed that the Hausdorff dimension of $\mathcal{D}_{k}$,

$$
\operatorname{dim}_{H} \mathcal{D}_{k}=\operatorname{dim} \mathcal{M}_{k}-\frac{1}{2}
$$

\footnotetext{
* Supported in part by a Postdoctoral Fellowship at MSRI.
} 
In 2, Cheung established some general strategy to get lower bound and upper bound of Hausdorff dimension. To compute the Hausdorff dimension of $\mathcal{D}_{k}$, Cheung made use of continued fractions to encode trajectories under the action of $A$, and a result on counting integer points in a particular region of $\mathbb{R}^{2}$, which he proved in 1 .

Because of this interesting result, it is natural to ask what happens if we replace $\mathbb{H}^{2}$ by $\mathbb{H}^{n}$ and replace the special lattice $\mathrm{SL}(2, \mathbb{Z})$ by other noncocompact lattices $\Gamma_{i}<\operatorname{Iso}\left(\mathbb{H}^{n}\right)=\mathrm{SO}(n, 1)$. To be precise, one could consider $k$ noncompact hyperbolic spaces $V_{i}=\Gamma_{i} \backslash \mathbb{H}^{n}$ where $\Gamma_{i}<\mathrm{SO}(n, 1)$ is a noncocompact lattice of $\mathrm{SO}(n, 1)$, i.e., $V_{i}$ is not compact and has finite volume. We define $\mathcal{M}_{k}:=\mathrm{T}^{1}\left(V_{1}\right) \times \mathrm{T}^{1}\left(V_{2}\right) \times \cdots \times \mathrm{T}^{1}\left(V_{k}\right)$, and consider the diagonal geodesic flow $g_{t} \times g_{t} \times \cdots \times g_{t}$ on $\mathcal{M}_{k}$ defined the same as above. Let $\mathcal{D}_{k}$ denotes the set of points with divergent forward trajectories, one could ask what the Hausdorff dimension of $\mathcal{D}_{k}$ is.

In this article we extend Cheung's work as follows:

Theorem 1.1. Let $k \geq 2$ and $V_{1}, V_{2}, \ldots, V_{k}$ be $k$ non-compact finite volume hyperbolic spaces of dimension $n$ and

$$
\mathcal{M}_{k}:=\mathrm{T}^{1}\left(V_{1}\right) \times \cdots \times \mathrm{T}^{1}\left(V_{k}\right)
$$

with diagonal geodesic flow $g_{t}: \mathcal{M}_{k} \rightarrow \mathcal{M}_{k}$ described as before. Denote

$$
\mathcal{D}_{k}:=\left\{\mathfrak{m} \in \mathcal{M}_{k}: g_{t}\left(\mathfrak{m}_{k}\right) \text { diverges as } t \rightarrow \infty\right\},
$$

then its Hausdorff dimension $\operatorname{dim}_{H} \mathcal{D}_{k}=k(2 n-1)-\frac{n-1}{2}$.

This work can be regarded as a part of a large program of studying the behavior of trajectories under diagonal flow and calculation of Hausdorff dimension of trajectories with certain properties in various dynamical systems. In 11, Kleinbock and Margulis studied bounded trajectories on homogeneous spaces under nonquasiunipotent flows and proved that the set of bounded trajectories has full Hausdorff dimension, although it has zero Lebesgue measure. The calculation makes use of the mixing property of nonquasiunipotent flows which is also the main tool of this article. As per divergent trajectories, Yitwah Cheung studied the trajectories on the homogeneous space $\mathrm{SL}(3, \mathbb{R}) / \mathrm{SL}(3, \mathbb{Z})$ under the diagonal flow $a(t):=\operatorname{diag}\left\{e^{t}, e^{t}, e^{-2 t}\right\}$ and proved that the Hausdorff dimension of set of divergent trajectories is $\operatorname{dim}(\mathrm{SL}(3, \mathbb{R}) / \mathrm{SL}(3, \mathbb{Z}))-\frac{2}{3}$, i.e., the Hausdorff co-dimension is equal to $\frac{2}{3}$ (see $\left.[3]\right)$. This result was recently extended to the space $\mathrm{SL}(d+1, \mathbb{R}) / \mathrm{SL}(d+1, \mathbb{Z})$ with diagonal flow $\operatorname{diag}\left\{e^{t}, e^{t}, \ldots, e^{t}, e^{-d t}\right\}$ for arbitrary $d \geq 2$ by Cheung and Chevallier (see [4]). If we do not restrict our attention on homogeneous spaces, we will find that the geodesic flows on translation surfaces share many properties in common. In [1], Cheung showed that the Hausdorff dimension of nonergodic direnctions of some particular translation surface is equal to $1 / 2$, with main ideas similar to that of [2]. This work was later extended by Cheung, P. Hubert and H. Masur in 5. The work in this direction can date back to the work of Masur and Smillie (see [14) and that of Masur (see [13]).

The basic idea to compute $\operatorname{dim}_{H} \mathcal{D}_{k}$ goes as follows:

For a non-compact finite volume $n$-dimensional hyperbolic space $V \cong \Gamma \backslash \mathbb{H}^{n}$, we focus our attention to the set of cusp points with respect to $\Gamma$ on the ideal boundary sphere $\partial \mathbb{H}^{n} \cong \mathbb{S}^{n-1}$ of $\mathbb{H}^{n}$, each of which corresponds to an infinite end of a fundamental domain of $V$. To study a particular geodesic ray $\mathcal{G}$ in $V$, it suffices to consider one of its lifts $\tilde{\mathcal{G}}$ in $\mathbb{H}^{n}$, then at some moment $\mathcal{G}$ is "near $\infty$ " in $V$ if and only if $\tilde{\mathcal{G}}$ is "close to" some cusp point on $\partial \mathbb{H}^{n}$, this associates every geodesic ray with a sequence of cusp points, and the time $\tilde{\mathcal{G}}$ stays near a cusp point can be estimated by some quantity called the height of the cusp point.

To get the lower bound of the Hausdorff dimension of $\mathcal{D}_{k}$, it suffices to consider the case when $k=2$ (since if a trajectory has divergent projection on the first two components, then itself is also divergent). On the first component, we choose the selection of geodesic rays such that the height of next cusp point is "much larger" than the preceding one, namely, the associate sequence of cusp points $\left\{\mathfrak{a}_{k}: k \in \mathbb{N}\right\}$ satisfies that for any $k \in \mathbb{N}, h\left(\mathfrak{a}_{k+1}\right) \asymp h^{1+\delta}\left(\mathfrak{a}_{k}\right)$, here $h(\cdot)$ denotes the height of the cusp point, and $\delta>0$ denotes some small constant. For each such geodesic ray $\mathcal{G}$ associated with $\left\{\mathfrak{a}_{k}: k \in \mathbb{N}\right\}$ on the first component, we choose the set of geodesics on the second component whose heights of corresponding cusp points all stay "far away" from each $h\left(\mathfrak{a}_{k}\right)$, i.e., no height lies in the interval $\left[\frac{h\left(\mathfrak{a}_{k}\right)}{\log h\left(\mathfrak{a}_{k}\right)}, h\left(\mathfrak{a}_{k}\right) \log h\left(\mathfrak{a}_{k}\right)\right]$ for all $k \in \mathbb{N}$. Every pair chosen as above gives a divergent trajectory on the product space, and the choice of the first component gives Hausdorff dimension $\frac{n-1}{2}$, and the second component contributes full Hausdorff dimension, this gives the lower bound of the Hausdorff dimension. 
As per the upper bound of the Hausdorff dimension, we firstly choose a compact subset $\mathcal{K}_{\rho} \subset \mathcal{M}_{k}$ depending on some parameter $\rho>0$, and define

$$
E_{k}(\rho):=\left\{\mathfrak{m} \in \mathcal{M}_{k}: g_{t}(\mathfrak{m}) \notin \mathcal{K}_{\rho} \text { for all large } t\right\},
$$

and then construct a so called self-similar covering of $E_{k}(\rho)$, and then apply the inequality proved in [2] to find the upper bound. This upper should depend on the parameter $\rho$, by letting $\rho \rightarrow 0$, we will get the same upper bound as the lower bound.

Compared with the work of Cheung ( [2]), the new ingredients of this article are as follows:

At first, in the work of Cheung, one only considers the special hyperbolic surface $\mathrm{SL}(2, \mathbb{Z}) \backslash \mathrm{SL}(2, \mathbb{R})$. In this case, the set of cusp points is the set of rational points (including $\infty$ ) on $\partial \mathbb{H}^{2} \cong \mathbb{R} \cup\{\infty\}$, and the height of a rational point $\frac{p}{q}$ ( $p$ and $q>0$ are coprime) is naturally its denominator $q$. But in general case, one needs to define the height of a cusp point properly so that it has most of the nice properties of the denominator of a rational number. This work is done in the third section of the article.

Secondly, as we have mentioned above, to get the lower bound of the Hausdorff dimension of $\mathcal{D}_{k}$, one needs to fix a geodesic ray $\mathcal{G}$ in the first component with property given above, with this fixed geodesic ray, one needs to choose the geodesic rays on the second component with heights far away from the heights of cusp points of $\mathcal{G}$. To compute the Hausdorff dimension of the geodesic rays on the second component, we will deal with the following counting problem on cusp points:

Problem 1.2. Given a cusp point $\mathfrak{a} \in \mathbb{R}^{n-1}$, and some large number $t>0$, how many cusp points $\mathfrak{b}$ satisfy that $h(\mathfrak{b}) \in\left[e^{t} h(\mathfrak{a}), 2 e^{t} h(\mathfrak{a})\right]$ and $\|\mathfrak{b}-\mathfrak{a}\| \leq \frac{1}{h(\mathfrak{a})}$ ? Here $\|\cdot\|$ denotes the Euclidean norm on $\mathbb{R}^{n-1}$.

In 2], the above counting problem is tailored to the following counting problems on rational numbers:

Problem 1.3. Given $d>0$ small and $h>0$ large, such that $h d$ is small enough but $h^{2} d$ is large enough, $x \in \mathbb{R}$ is some real number such that there is a convergent $\frac{p}{q}$ of $x$ satisfying $(h d)^{-1} \leq q \leq h$, then how many reduced rational numbers $\frac{p^{\prime}}{q^{\prime}}$ in the interval $[x-d, x+d]$ satisfy that $q^{\prime} \in[h, 2 h]$ ?

This question was answered in another work [1. In that work, the counting problem on rational numbers was reduced to counting problem on integer points in $\mathbb{Z}^{2}$ inside a particular region of $\mathbb{R}^{2}$. The counting was done via careful study of integer points in $\mathbb{R}^{2}$.

But this argument could not be modified to solve the above general counting problem, the correspondence between cusp points in $\mathbb{R}$ and lattice points in $\mathbb{R}^{2}$ only exists for $\Gamma=\operatorname{SL}(2, \mathbb{Z})$. For general case, a new approach is needed.

It turns out that by making use of the mixing property of geodesic flow on $\mathrm{T}^{1}(V)$, the counting can be done. The detail will be discussed in the fourth section.

The result of this article is possible to extend in the following directions:

Problem 1.4. One can drop the finite volume condition of the hyperbolic spaces, and instead, assume that every component $V_{i}$ is geometrically finite. In this case, one can define $\mathcal{D}_{k}$ as follows:

$$
\mathcal{D}_{k}:=\left\{\left(v_{1}, \ldots, v_{k}\right) \in \mathcal{M}_{k}: g_{t}\left(v_{1}, \ldots, v_{k}\right) \text { diverges but } g_{t}\left(v_{i}\right) \text { does not diverges for each } i\right\} \text {. }
$$

And ask what is the Hausdorff dimension of $\mathcal{D}_{k}$.

Remark 1.5. We add the condition that $g_{t}\left(v_{i}\right)$ does not diverge for each $i$ because if $V_{i}$ has infinite volume, the set of divergent trajectories on each $V_{i}$ has full Hausdorff dimension (actually, it has positive Lebesgue measure), the problem will be trivial without the additional condition.

The article is organized as follows:

- In the second section, we will recall some basic theory of Lie groups and hyperbolic spaces, and make a basic reduction of the original problem.

- In the third section, we discuss the structure of a general finite volume hyperbolic space $V=\Gamma \backslash \mathbb{H}^{n}$, general properties of cusp points on the ideal infinite boundary $\partial \mathbb{H}^{n}$ and basic properties of geodesic rays on $V$.

- In the fourth section, we will prove the counting result on cusp points mentioned above. This is the most important technical result for getting the lower bound of Hausdorff dimension of $\mathcal{D}_{k}$. 
- In the fifth section, we will finish the computation of Hausdorff dimension, the first part will give the lower bound, and the second part will give the upper bound.

Notations 1.6. We will use the following notations: for two quantities $A$ and $B$, we will use $A \ll B$ to mean that there is a constant $C>0$, only depending on the structure of $\Gamma_{i}$ 's, such that $A \leq C B$, we use $A \gg B$ to mean that $B \ll A$, and use $A \asymp B$ to mean that $A \ll B$ and $B \ll A$.

Acknowledgement: This article is part of my thesis, I would like to express my deep gratitude to my advisor, Professor Nimish Shah, for his immensurable amount of support and guidance during the process of this work. I also would like to thank Dmitry Kleinbock and Yitwah Cheung for reading an earlier version of this paper and giving a lot of comments and suggestions. Thanks are also due to the referees for many useful suggestions.

\section{Prelimenaries on hyperbolic spaces AND Basic Reduction}

In this section, we recall some basic theory of Lie groups and hyperbolic spaces, and reduce the original problem to a relatively simple problem.

Let $V$ be some non-compact hyperbolic space of dimension $n$ with finite total volume, then we have $V=\Gamma \backslash \mathbb{H}^{n}$, where $\mathbb{H}^{n}$ is the universal $n$-dimensional hyperbolic space and $\Gamma=\pi_{1}(V)$. It is well known that $\mathrm{Iso}\left(\mathbb{H}^{n}\right) \cong \mathrm{SO}(n, 1)$, in this article, we denote it by $G$. For $x \in \mathbb{H}^{n}$, the group of stabilizers of $x$ in $G$ is $K \cong \mathrm{SO}(n)$, so $\mathbb{H}^{n} \cong G / K$, and for $v \in \mathrm{T}^{1}\left(\mathbb{H}^{n}\right)$, the group of stabilizers of $v$ in $G$ is $M \cong \mathrm{SO}(n-1)$, so the unit tangent bundle $\mathrm{T}^{1}\left(\mathbb{H}^{n}\right) \cong G / M$. And $\Gamma$ can be identified with a discrete subgroup of $G$, such that $\Gamma \backslash G$ admits a finite measure invariant under the right multiplication of $G$. We denote the Lie algebra of $G$ by $\mathfrak{g}=\mathfrak{s o}(n, 1)$, according to the theory of Lie groups and Lie algebras, $G$ admits a one-dimensional maximal $\mathbb{R}$-split torus $A$ and a characteristic $\lambda: A \rightarrow \mathbb{R}_{+}$such that $\mathfrak{g}$ decomposes as follows according to the adjoint action of $A$ :

$$
\mathfrak{g}=\mathfrak{g}_{-1} \oplus \mathfrak{z}(A) \oplus \mathfrak{g}_{+1}
$$

where $\mathfrak{z}(A)$ is the Lie algebra of the centralizer $Z(A) \cong M A$ of $A$, and

$$
\mathfrak{g}_{ \pm 1}=\left\{v \in \mathfrak{g}: \operatorname{Ad}(a) v=\lambda(a)^{ \pm 1} v \text { for any } a \in A\right\} .
$$

We parametrize $A=\{a(t): t \in \mathbb{R}\}$ such that $\lambda(a(t))=e^{t}$.

The Weyl group element $\sigma$ with respect to the torus $A$ has a representative in $K$, which we also denote by $\sigma$. Then $\sigma^{2}=\mathrm{id}$ and $\sigma a(t) \sigma^{-1}=a(-t)$.

It is well known that $\mathfrak{g}_{+1} \cong \mathbb{R}^{n-1}$, and $\mathfrak{g}_{+}$is the Lie algebra of the expanding horospherical subgroup $N$ with respect to the conjugate action of $A$. We identify $\mathfrak{g}_{+}$by $\mathbb{R}^{n-1}$ and parametrize $N$ by

$$
N=\left\{u(\mathbf{x})=\exp (\mathbf{x}): \mathbf{x} \in \mathbb{R}^{n-1} \cong \mathfrak{g}_{+1}\right\} .
$$

Similarly, $\mathfrak{g}_{-1} \cong \mathbb{R}^{n-1}$ is the Lie algebra of the contracting horospherical subgroup $U^{-}$with respect to the conjugate action of $A$. Because $U^{-}=\sigma N \sigma$, we could parametrize $U^{-}$by

$$
U^{-}=\left\{u^{-}(\mathbf{x})=\sigma u(\mathbf{x}) \sigma: \mathbf{x} \in \mathbb{R}^{n-1}\right\} .
$$

In this article, we will fix a Euclidean norm $\|\cdot\|$ on $\mathbb{R}^{n-1}$.

We have the following Iwasawa decomposition:

$$
\begin{aligned}
& N \times A \times K \rightarrow G \\
& (n, a, k) \mapsto n a k
\end{aligned}
$$

where the map is group multiplication, and it is a diffeomorphism.

It is also well known that

$$
\begin{aligned}
& N \times M A \times U^{-} \rightarrow G \\
& \left(u(\mathbf{x}), m a, u^{-}(\mathbf{y})\right) \mapsto u(\mathbf{x}) \operatorname{mau}^{-}(\mathbf{y})
\end{aligned}
$$

is a diffeomorphism.

Let $P=M A N$ denote a parabolic subgroup of $G$, we have the following Bruhat decomposition:

$$
G=P \cup N \sigma P
$$

Then the ideal boundary $\partial \mathbb{H}^{n} \cong G / P=P / P \cup N \sigma P / P$, we may identify $N \sigma P / P$ with $\mathbb{R}^{n-1}$ and denote $P / P$ by $\infty$. Then we have $\partial \mathbb{H}^{n}=\mathbb{R}^{n-1} \cup\{\infty\} \cong \mathbb{S}^{n-1}$. 
To study the homogeneous space $\Gamma \backslash G$, we need to know the shape of its fundamental domain, especially its shape near infinity.

Let $\eta \subset N$ be a compact subset of $N$, and for some $s \in \mathbb{R}$, denote

$$
A_{s}=\{a(t): t \geq s\} \subset A
$$

We define

$$
\Omega(\eta, s)=\eta A_{s} K
$$

Thanks to Garland and Raghunathan, we have the following result concerning the fundamental domain:

Theorem 2.1. (See [9, Theorem 0.6 and Theorem 0.7])

There exists $s_{0}>0$, a compact subset $\eta_{0}$ of $N$ and a finite subset $\Xi$ of $G$ such that

(1) $G=\Gamma \Xi \Omega\left(s_{0}, \eta_{0}\right)$

(2) for all $\xi \in \Xi$, the group $\Gamma \cap \xi N \xi^{-1}$ is a cocompact lattice in $\xi N \xi^{-1}$

(3) for all compact subset $\eta$ of $N$ the set

$$
\left\{\gamma \in \Gamma: \gamma \Xi \Omega\left(s_{0}, \eta\right) \cap \Omega\left(s_{0}, \eta\right) \neq \emptyset\right\}
$$

is finite

(4) for each compact subset $\eta$ of $N$ containing $\eta_{0}$ there exists $s_{1}>s_{0}$ such that for all $\xi_{1}, \xi_{2} \in \Xi$ and $\gamma \in \Gamma$ with $\gamma \xi_{1} \Omega\left(s_{0}, \eta\right) \cap \xi_{2} \Omega(s, \eta) \neq \emptyset$, we have $\xi_{1}=\xi_{2}$ and $\gamma \in \xi_{1} N M \xi_{1}^{-1}$

Without loss of generality, we may assume $e \in \Xi$, since otherwise we can replace $\Gamma$ with some conjugate $\xi^{-1} \Gamma \xi$ to move $\xi \in \Xi$ to $e$. Therefore we can define the set of cusp points of $\Gamma \backslash \mathbb{H}^{n}$ in the ideal boundary $\partial \mathbb{H}^{n}=\mathbb{R}^{n-1} \cup\{\infty\}$ to be $\Gamma \backslash \mathbb{H}^{n}$ to be $\Gamma \Xi \infty \subset \mathbb{R}^{n-1} \cup\{\infty\}$. Our assumption that $e \in \Xi$ is equivalent to saying that $\infty$ is a cusp of $\Gamma \backslash \mathbb{H}^{n}$. It is easy to see that $\Gamma x a(t) M$ diverges as $t \rightarrow \infty$ if $x a(t) P / P \in \partial \mathbb{H}^{n}$ is a cusp of $\Gamma \backslash \mathbb{H}^{n}$.

Then for any $v \in \mathrm{T}^{1}(V) \cong \Gamma \backslash G / M$, we can represent $v$ by $\Gamma x M$ for some $x \in G$, then the geodesic flow is just the group action of $A$, to be precise, $g_{t}(\Gamma x M)=\Gamma x a(t) M$. Note that $M \subset Z(A)$.

Then for a product of $k$ such spaces $\Gamma_{1} \backslash G / M \times \cdots \times \Gamma_{k} \backslash G / M$, for the argument above, we may assume that $\infty$ is a cusp of each $\Gamma_{i} \backslash \mathbb{H}^{n}$. Then for a general point $\left(\Gamma_{1} x_{1} M, \ldots, \Gamma_{k} x_{k} M\right) \in \Gamma_{1} \backslash G / M \times \cdots \times \Gamma_{k} \backslash G / M$, if any $\Gamma_{i} x_{i}$ has a representative in $P=M A N$, we may assume that $x_{i} \in P$ then $x_{i} a(t) P / P=\infty$, since $\infty$ is a cusp of $\Gamma_{i} \backslash \mathbb{H}$, we have $\Gamma_{i} x_{i} a(t) M$ diverges in $\Gamma_{i} \backslash G / M$ as $t \rightarrow \infty$, and thus $\left(\Gamma_{1} x_{1} a(t) M, \cdots, \Gamma_{k} x_{k} a(t) M\right)$ diverges in the product space as $t \rightarrow \infty$. The set of such trajectories has Hausdorff codimension $n-1$. Now we assume every $x_{i}$ is of form $n_{i} \sigma p_{i}$, where $n_{i} \in N$ and $p_{i} \in P$, Then we have $\left(\Gamma_{1} x_{1} a(t) M, \ldots, \Gamma_{k} x_{k} a(t) M\right)=$ $\left(\Gamma_{1} n_{1} \sigma a(t) a(-t) p_{1} a(t) M, \ldots, \Gamma_{k} n_{k} \sigma a(t) a(-t) p_{k} a(t) M\right)$. Since for any $n \in N, a(-t) n a(t) \rightarrow e$ as $t \rightarrow \infty$, and $M A \subset Z(A)$, we have for any large $t, a(-t) p_{i} a(t)$ remains in some compact subset of $G$ depending on $p_{i}$, therefore, $\left(\Gamma_{1} x_{1} a(t) M, \ldots, \Gamma_{k} x_{k} a(t) M\right)$ diverges if and only if $\left(\Gamma_{1} n_{1} \sigma a(t) M, \ldots, \Gamma_{k} n_{k} \sigma a(t) M\right)$ diverges, as $t \rightarrow \infty$. Now we focus our attention to the geodesics of form $\{u(\mathbf{x}) \sigma a(t): t>0\}$ where $u(\mathbf{x}) \in N$ defined as above.

Define:

$$
\mathfrak{B}_{k}=\left\{\left(\mathbf{x}_{1}, \ldots, \mathbf{x}_{k}\right) \in\left(\mathbb{R}^{n-1}\right)^{k}:\left(\Gamma_{1} u\left(\mathbf{x}_{1}\right) \sigma a(t) M, \ldots, \Gamma_{k} u\left(\mathbf{x}_{k}\right) \sigma a(t) M\right) \text { diverges as } t \rightarrow \infty\right\}
$$

Then by the argument above and the property of Hausdorff dimension, we have

$$
\operatorname{dim}_{H} \mathfrak{B}_{k}=\operatorname{dim}_{H} \mathfrak{D}_{k}-k \operatorname{dim}(P / M)
$$

Since $\operatorname{dim}(P / M)=\operatorname{dim}(N A)=n$, we reduce the original problem to showing the following statement:

\section{Proposition 2.2.}

$$
\operatorname{dim}_{H} \mathfrak{B}_{k}=k(n-1)-\frac{n-1}{2} .
$$

\section{Geodesic Rays on hyperbolic space and Cusp points}

In this section, we will fix a hyperbolic non-compact hyperbolic space $V=\Gamma \backslash \mathbb{H}^{n}$ with finite volume, and discuss properties of geodesic rays on $V$ and cusp points of $\Gamma$ on $\partial \mathbb{H}^{n}$. Because of the reduction in the previous section, we only look at geodesic rays of form $\{\Gamma u(\mathbf{x}) \sigma a(t) M: t>0\}$.

By Theorem 2.1, a typical fundamental domain $\mathcal{F}$ of the action of $\Gamma$ on $\mathbb{H}^{n}$ is the union of a compact subset $\mathcal{K}$ and finitely infinite cusp of form $\gamma \xi_{i} \Omega\left(\eta, s_{1}\right) / K$, where $\xi_{i}$ runs over elements of $\Xi$. We can choose 
a fixed fundamental domain $\mathcal{F}_{0}$ such that $K \in \mathcal{F}_{0}$, and we may change $\Xi$ so that $\mathcal{F}_{0}$ is union of a compact subset and $\xi_{i} \Omega\left(\eta, s_{1}\right) / K$.

By our discussion in the previous section, every cusp point is of form $\gamma \xi \infty$, where $\gamma \in \Gamma$, and $\xi \in \Xi$.

Definition 3.1. We consider the Bruhat decomposition of $\gamma \xi=u(\mathbf{x}) \sigma u(\mathbf{y}) a(r) m$ where $m \in M$, then we define the height of the cusp point $\mathfrak{a}=\gamma \xi \infty$ to be $h(\mathfrak{a})=e^{r}$. It is easily seen that $\gamma \xi \in P$ iff and only $\gamma=\xi=e$, the corresponding cusp is $\infty$, we define the height of $\infty$ to be $h(\infty)=1$.

Remark 3.2. For $m \in M \cong \mathrm{SO}(n-1)$, and $\mathbf{x} \in \mathbb{R}^{n-1}$, the conjugation $m u(\mathbf{x}) m^{-2}=u(m \mathbf{x})$, where the action of $M \cong \mathrm{SO}(n-1)$ on $\mathbb{R}^{n-1}$ is the natural action. In particular, it preserves the norm $\|\cdot\|$. For a fixed cusp $\mathfrak{a}=\gamma \xi \infty$, the subgroup of $\Gamma$, denoted by $\Gamma_{\mathfrak{a}}$ is equal to $\gamma \Gamma_{\xi} \gamma^{-1}$, where $\Gamma_{\xi}=\Gamma \cap \xi N \xi^{-1}$, which is an unipotent subgroup. So we can replace $\gamma \xi \infty$ by $\gamma \xi u(\mathbf{n}) \infty$ where $u(\mathbf{n}) \in N \cap \xi^{-1} \Gamma \xi$. Then if $\|\mathbf{y}\| \gg e^{r}$ or $\|\mathbf{y}\| \ll e^{r}$, we may choose $\mathbf{n}$ appropriately such that $\left\|\mathbf{y}+e^{r} m \mathbf{n}\right\| \asymp e^{r}$, then

$$
\begin{aligned}
\gamma \xi u(\mathbf{n}) & =u(\mathbf{x}) \sigma u(\mathbf{y}) a(r) m u(\mathbf{n}) \\
& =u(\mathbf{x}) \sigma u\left(\mathbf{y}+e^{r} m \mathbf{n}\right) a(r) m .
\end{aligned}
$$

So we can assume that $\|\mathbf{y}\| \asymp e^{r}$.

Since $\mathbb{H}^{n}=G / K$, by Iwasawa decomposition, $G / K=N A K / K \cong N A$. We will use the coordinate system in [7, denoting $u(\mathbf{x}) a(t) K / K$ by $\left(e^{t}, \mathbf{x}\right)$. We call it the $N A$-coordinate system.

In [7, the action of Weyl group element $\sigma$ on $G / K$ is given as follows:

$$
\sigma\left(e^{t}, \mathbf{x}\right)=\sigma u(\mathbf{x}) a(t) K / K=\frac{1}{e^{2 t}+\|\mathbf{x}\|^{2}}\left(e^{t},-\mathbf{x}\right)
$$

We consider a typical geodesic ray $\mathcal{G}_{\mathbf{x}}=\left\{\mathcal{G}_{\mathbf{x}}(t): t>0\right\}\left(\right.$ where $\left.\mathbf{x} \in \mathbb{R}^{n-1}\right)$ as follows:

$$
\mathcal{G}_{\mathbf{x}}(t)=u(\mathbf{x}) \sigma a(t)
$$

Definition 3.3. Take $s_{1}>s_{0}>0$ as in Theorem 2.1, we say $\mathcal{G}_{\mathbf{x}}$ enters (or is near) the cusp $\mathfrak{a}=\gamma \xi \infty$ at $t$ if

$$
u(\mathbf{x}) \sigma a(t) \in \gamma \xi N A_{s_{1}} K .
$$

We say $\mathcal{G}_{\mathbf{x}}$ enters (or is near) the cusp $\mathfrak{a}=\gamma \xi \infty$, if it enters (or is near) $\mathfrak{a}$ at $t$ for some $t>0$.

For $\mathbf{x} \in \mathbb{R}^{n-1}$, we define the spectrum of $\mathbf{x}$, denoted by $\operatorname{Spec}(\mathbf{x})$ to be the sequence $\left\{\mathfrak{a}_{i}: i \in \mathbb{N}\right\}$ of cusp points $\mathcal{G}_{\mathbf{x}}$ enters, ordered by the time $t_{i}$ at which $\mathcal{G}_{\mathbf{x}}$ enters $\mathfrak{a}_{i}$.

Concerning the height of cusp point and geodesic rays near the cusp, we have the following proposition similar to rational convergents of real numbers:

Proposition 3.4. If $\mathcal{G}_{\mathbf{x}}$ enters a cusp $\mathfrak{a}=\gamma \xi \infty$, then

$$
\|\mathbf{x}-\mathfrak{a}\| \ll \frac{1}{h(\mathfrak{a})} .
$$

Here $\|\cdot\|$ denotes the Euclidean norm on $\mathbb{R}^{n-1}$. Conversely, there exists a constant $c>0$ such that if

$$
\|\mathbf{x}-\mathfrak{a}\| \leq \frac{c}{h(\mathfrak{a})}
$$

then $\mathcal{G}_{\mathbf{x}}$ enters $\mathfrak{a}$.

Remark 3.5. In the case $n=2$ and $\Gamma=\mathrm{SL}(2, \mathbb{Z})$, the cusp points are rationals, and the height of a reduced rational $\frac{p}{q}((p, q)=1)$ is $q^{2}$, and for $x \in \mathbb{R}$, a geodesic ray $\mathcal{G}_{x}$ enters $\frac{p}{q}$ if and only if $\frac{p}{q}$ is a convergent of $x$, which is equivalent to the above inequality holds. Thus the above proposition extends this approximations of rationals to any dimension $n \geq 2$ and any lattice $\Gamma<\mathrm{SO}(n, 1)$.

Proof. Suppose at $t_{0}, \mathcal{G}_{\mathbf{x}}$ enters $\mathfrak{a}=\gamma \xi \infty$, then $u(\mathbf{x}) \sigma a\left(t_{0}\right)=\gamma \xi n a(s) k$, where $e^{s} \asymp e^{s_{0}} \asymp 1$. Write $\gamma \xi=u\left(\mathbf{x}_{1}\right) \sigma u\left(\mathbf{x}_{2}\right) a(r) m$ in the Bruhat decomposition. Then

$$
u(\mathbf{x}) \sigma a\left(t_{0}\right)=u\left(\mathbf{x}_{1}\right) \sigma u\left(\mathbf{x}_{2}\right) a(r+s) k^{\prime},
$$

where $k^{\prime}=k m \in K$. Rewrite it as follows:

$$
a(-r) u\left(-\mathbf{x}_{2}\right) \sigma u\left(\mathbf{x}-\mathbf{x}_{1}\right) \sigma a\left(t_{0}\right)=a(s) k^{\prime}
$$


Put both sides into the $N A$-coordinate system, then

$$
\operatorname{LHS}=\left(\frac{e^{-t_{0}-r}}{e^{-2 t_{0}}+\left\|\mathbf{x}-\mathbf{x}_{1}\right\|^{2}}, e^{-r}\left(\frac{\mathbf{x}_{1}-\mathbf{x}}{e^{-2 t_{0}}+\left\|\mathbf{x}-\mathbf{x}_{1}\right\|^{2}}-\mathbf{x}_{2}\right)\right)
$$

and

$$
\operatorname{RHS}=\left(e^{s}, \mathbf{0}\right)
$$

Then $1 \asymp e^{s}=\frac{e^{-t_{0}-r}}{e^{-2 t_{0}}+\left\|\mathbf{x}-\mathbf{x}_{1}\right\|^{2}}$. We denote

$$
\begin{aligned}
f\left(t_{0}\right) & =\frac{e^{-t_{0}-r}}{e^{-2 t_{0}+\left\|\mathbf{x}-\mathbf{x}_{1}\right\|^{2}}} \\
& =\frac{e^{-r}}{e^{-t_{0}}+e^{t_{0}}\left\|\mathbf{x}-\mathbf{x}_{1}\right\|^{2}},
\end{aligned}
$$

$f\left(t_{0}\right)$ is increasing when $t_{0} \leq\left\|\mathbf{x}-\mathbf{x}_{1}\right\|^{-1}$ and decreasing when $t_{0}>\left\|\mathbf{x}-\mathbf{x}_{1}\right\|^{-1}$. The maximum of $f\left(t_{0}\right)$ is equal to $\frac{e^{-r}}{2\left\|\mathbf{x}-\mathbf{x}_{1}\right\|}$, so $1 \asymp f\left(t_{0}\right)$ implies that

$$
\frac{e^{-r}}{2\left\|\mathbf{x}-\mathbf{x}_{1}\right\|} \gg 1
$$

Note that $h(\mathfrak{a})=e^{r}$ and $\mathfrak{a}=\mathbf{x}_{1}$ (because $\left.\mathfrak{a}=\gamma \xi \infty \in u\left(\mathbf{x}_{1}\right) \sigma P\right)$, then the above inequality completes the first part of the proof.

Conversely, if the maximum of $f\left(t_{0}\right)$, say $\frac{e^{r}}{2\left\|\mathbf{x}-\mathbf{x}_{1}\right\|}$ is large enough, we could make $e^{s} \geq e^{s_{1}}$ for some $t_{0}$, in particular, $\mathcal{G}_{\mathbf{x}}\left(t_{0}\right)$ is near $\mathfrak{a}$. This proves the second part.

Remark 3.6. From the proof above, we note that the function $f\left(t_{0}\right)$ describes how deep a geodesic ray $\mathcal{G}_{\mathbf{x}}\left(t_{0}\right)$ enters into a cusp $\mathfrak{a}$ : when $f\left(t_{0}\right)$ is large the geodesic ray is near the cusp.

Definition 3.7. Given a geodesic ray $\mathcal{G}_{\mathbf{x}}$ and a cusp point $\mathfrak{a}$, we define the depth function $f_{\mathfrak{a}}(t, \mathbf{x})$ with respect to $\mathfrak{a}$ as follows:

$$
f_{\mathfrak{a}}(t, \mathbf{x})=\frac{1}{h(\mathfrak{a})\left(e^{-t}+e^{t}\|\mathbf{x}-\mathfrak{a}\|^{2}\right)} .
$$

We define the total depth function $W(t, \mathbf{x})$ as follows:

$$
W(t, \mathbf{x})=\max _{\mathfrak{a} \in \Gamma \Xi \infty}\left\{f_{\mathfrak{a}}(t, \mathbf{x})\right\} .
$$

Corollary 3.8. Given $\mathbf{x} \in \mathbb{R}^{n-1}$ and a cusp point $\mathfrak{a}$ such that $\|\mathbf{x}-\mathfrak{a}\| h(\mathfrak{a})$ is small enough, then the time $t_{1}$ when $\mathcal{G}_{\mathbf{x}}$ enters $\mathfrak{a}$ satisfies:

$$
e^{t_{1}} \asymp h(\mathfrak{a}),
$$

the time $\tau$ when $f_{\mathfrak{a}}(t)$ has its maximum satisfies:

$$
e^{\tau}=\frac{1}{\|\mathbf{x}-\mathfrak{a}\|}
$$

and the time $t_{2}$ when $\mathcal{G}_{\mathbf{x}}$ leaves $\mathfrak{a}$ satisfies:

$$
e^{t_{2}} \asymp \frac{1}{h(\mathfrak{a})\|\mathbf{x}-\mathfrak{a}\|^{2}} .
$$

Proof. We consider the function $f(t)=f_{\mathfrak{a}}(t, \mathbf{x})$. It admits its unique maximum $\frac{1}{h(\mathfrak{a})\|\mathbf{x}-\mathfrak{a}\|}$ at $t=-\log (\| \mathbf{x}-$ $\mathfrak{a} \|):=\tau$. This proves the second equation.

When $t<\tau$, since $e^{-t}>e^{t}\|\mathbf{x}-\mathfrak{a}\|^{2}$,

$$
f(t) \asymp \frac{1}{h(\mathfrak{a}) e^{-t_{0}}} .
$$

Suppose at $t=t_{1}, \mathcal{G}_{\mathbf{x}}$ enters $\mathfrak{a}$, then $t_{1}<\tau$, and $f\left(t_{1}\right) \asymp 1$, this shows that

$$
e^{t_{1}} \asymp h(\mathfrak{a}) .
$$

When $t>\tau$, since $e^{-t}<e^{t}\|\mathbf{x}-\mathfrak{a}\|^{2}$,

$$
f(t) \asymp \frac{1}{h(\mathfrak{a})\|\mathbf{x}-\mathfrak{a}\|^{2} e^{t}} .
$$


Suppose at $t=t_{2}, \mathcal{G}_{\mathbf{x}}$ leaves $\mathfrak{a}$, then $t_{2}>\tau$, and $f(t) \asymp 1$, this shows that

$$
e^{t_{2}} \asymp \frac{1}{h(\mathfrak{a})\|\mathbf{x}-\mathfrak{a}\|^{2}} .
$$

This completes the proof.

Remark 3.9. For $\mathbf{x} \in \mathbb{R}^{n-1}$, we have defined its spectrum

$$
\operatorname{Spec}(\mathbf{x})=\{\mathfrak{a}(i, \mathbf{x}): i \in \mathbb{N}\}
$$

we denote by $t_{1}(i, \mathbf{x}), \tau(i, \mathbf{x})$ and $t_{2}(i, \mathbf{x})$ the times when $\mathcal{G}_{\mathbf{x}}$ enters $\mathfrak{a}(i, \mathbf{x})$, when $f_{\mathfrak{a}(i, \mathbf{x})}(t, \mathbf{x})$ admits its maximum, and when $\mathcal{G}_{\mathbf{x}}$ leaves $\mathfrak{a}(i, \mathbf{x})$, respectively. Then according the above proposition,

and

$$
\begin{gathered}
e^{t_{1}(i, \mathbf{x})} \asymp h(\mathfrak{a}(i, \mathbf{x})), \\
e^{\tau(i, \mathbf{x})}=\frac{1}{\|\mathbf{x}-\mathfrak{a}(i, \mathbf{x})\|},
\end{gathered}
$$

$$
e^{t_{2}(i, \mathbf{x})} \asymp \frac{1}{h(\mathfrak{a}(i, \mathbf{x}))\|\mathbf{x}-\mathfrak{a}(i, \mathbf{x})\|^{2}} .
$$

When a geodesic ray $\mathcal{G}_{\mathbf{x}}$ is in the compact part of some fundamental domain, we can still associate it to a cusp point of this fundamental domain, under this condition, we say the geodesic ray is roughly near the cusp point:

Definition 3.10. We can enlarge the cusp parts of $\Gamma \backslash \mathbb{H}^{n}$ such that their union covers the whole space. Then in the space $\mathbb{H}^{n}$, we can divide each fundamental domain into several enlarged cusp parts such that they cover the whole $\mathbb{H}^{n}$. For $\mathbf{x} \in \mathbb{R}^{n-1}$, we say that the geodesic ray $\mathcal{G}_{\mathbf{x}}$ roughly enters a cusp $\mathfrak{a}$ (or is roughly near the cusp $\mathfrak{a}$ ) at time $t$ if $\mathcal{G}_{\mathbf{x}}(t)$ is inside the enlarged cusp part associated with $\mathfrak{a}$. In this sense, we can define the rough spectrum of $\mathbf{x}$ as the sequence of cusps that $\mathcal{G}_{\mathbf{x}}$ roughly enters.

Remark 3.11. Using the same argument as the proof of Proposition 3.4, it is easily shown that:

Corollary 3.12. if $\mathcal{G}_{\mathbf{x}}$ roughly enters a cusp point $\mathfrak{a}$, then

$$
\|\mathbf{x}-\mathfrak{a}\| \ll \frac{1}{h(\mathfrak{a})} .
$$

Moreover, following the argument in the proof of Corollary 3.8, we can deduce the same result concerning the time $\mathcal{G}_{\mathbf{x}}$ roughly enters and leaves a cusp point:

Corollary 3.13. For $\mathbf{x} \in \mathbb{R}^{n-1}$, suppose the associated geodesic ray $\mathcal{G}_{\mathbf{x}}$ roughly enters a cusp point $\mathfrak{a}$, then the time $t_{1}$ when $\mathcal{G}_{\mathbf{x}}$ roughly enters $\mathfrak{a}$ and the time $t_{2}$ when $\mathcal{G}_{\mathbf{x}}$ roughly leaves $\mathfrak{a}$ satisfy

$$
e^{t_{1}} \asymp h(\mathfrak{a}),
$$

and

respectively.

$$
e^{t_{2}} \asymp \frac{1}{h(\mathfrak{a})\|\mathbf{x}-\mathfrak{a}\|^{2}}
$$

Now we will define an alternative metric $d_{o}(\cdot, \cdot)$ (with respect to a base point $o \in \mathcal{F}_{0}$ ) and the height of a cusp point $\tilde{h}(\mathfrak{a})$, and prove that in a fixed ball $B \subset \mathbb{R}^{n-1}, h(\mathfrak{a}) \asymp \tilde{h}(\mathfrak{a})$ and $\|\mathbf{x}-\mathbf{y}\| \asymp d_{o}(\mathbf{x}, \mathbf{y})$. These definitions naturally come from the geometric structure of hyperbolic spaces and can be generalized to any Riemannian manifold with negative curvature.

We firstly recall the basic theory of Busemann function.

For $\xi \in \partial \mathbb{H}^{n}$ and $x, y \in \mathbb{H}^{n}, B_{\xi}(x, y)$ is defined as follows:

$$
B_{\xi}(x, y)=\lim _{t \rightarrow \infty} d_{\mathbb{H}}(x, \xi(t))-d_{\mathbb{H}}(y, \xi(t)),
$$

where $d_{\mathbb{H}}$ denotes the hyperbolic distance in $\mathbb{H}^{n}$ and $\{\xi(t)\}_{t \geq 0}$ is any geodesic ray pointing to $\xi$. It turns out that the value is independent of the choice of the ray $\{\xi(t)\}$.

For $B_{\xi}(x, y)$ we have the following basic properties: 
(1) Let $\{\xi(t)\}_{t \geq 0}$ be a geodesic ray pointing to $\xi$, then we have $B_{\xi}\left(\xi\left(t_{1}\right), \xi\left(t_{2}\right)\right)=t_{2}-t_{1}$ for any nonnegative numbers $t_{1}, t_{2}$.

(2) If $y=n_{\xi} x$, for some $n_{\xi} \in \mathcal{U}(\xi)=\{g \in G: g$ is unipotent and $g \xi=\xi\}$, then $B_{\xi}(x, y)=0$.

(3) $B_{\xi}(x, y)+B_{\xi}(y, z)=B_{\xi}(x, z), B_{\xi}(x, y)=-B_{\xi}(y, x)$.

(4) For any $g \in G$ we have $B_{g \xi}(g x, g y)=B_{\xi}(x, y)$.

Definition 3.14. Fix $o=K \in \mathcal{F}_{0}$. For a cusp $\mathfrak{a}=\gamma \xi \infty$,

$$
\tilde{h}(\mathfrak{a})=\exp \left(B_{\xi \infty}\left(\gamma^{-1} o, o\right)\right) .
$$

Definition 3.15. For two points $\xi_{1}, \xi_{2} \in \partial \mathbb{H}^{n}$, and any point $x \in \mathbb{H}^{n}$, we define the Gromov metric between $\xi_{1}$ and $\xi_{2}$ respect to $x$ (denoted by $\left.d_{x}\left(\xi_{1}, \xi_{2}\right)\right)$ as follows:

$$
d_{x}\left(\xi_{1}, \xi_{2}\right)=\exp \left(-\frac{1}{2}\left(\lim _{t \rightarrow \infty} B_{\xi_{1}}(x, \xi(t))+B_{\xi_{2}}(x, \xi(t))\right)\right),
$$

where $\{\xi(t): t \geq 0\}$ denotes any geodesic ray pointing to any point $\xi$ at infinity. The value is independent of the choice of $\{\xi(t): t \geq 0\}$.

Remark 3.16. An important property of the Gromov metric is that it is uniformly bounded, namely, there exists a constant $M>0$ such that

$$
d_{o}\left(\xi_{1}, \xi_{2}\right) \leq M
$$

for all $\xi_{1}, \xi_{2} \in \partial \mathbb{H}^{n}$.

Proposition 3.17. Given a fixed compact subset $B \subset \mathbb{R}^{n-1}$, for any $\mathbf{x}, \mathbf{y} \in B$,

$$
d_{o}(\mathbf{x}, \mathbf{y}) \asymp\|\mathbf{x}-\mathbf{y}\|,
$$

and for any cusp point $\mathfrak{a} \in B$,

$$
\tilde{h}(\mathfrak{a}) \asymp h(\mathfrak{a}) .
$$

Proof. For $\mathbf{x}, \mathbf{y} \in B$, choose $k, k^{\prime} \in K$ such that $k a(t) \rightarrow$ mathbf $x$ and $k^{\prime} a(t) \rightarrow \mathbf{y}$ as $t \rightarrow \infty$. We could choose $k=u(\mathbf{x}) \sigma n_{1} a\left(t_{1}\right)$ and $k^{\prime}=u(\mathbf{y}) \sigma n_{2} a\left(t_{2}\right)$. Then from $\mathbf{x}, \mathbf{y} \in B$, it is easily seen that $n_{1}, n_{2}$ and $a\left(t_{1}\right), a\left(t_{2}\right)$ are uniformly bounded, in particular, $e^{t_{1}} \asymp e^{t_{2}} \asymp 1$. Choose the geodesic ray $\left\{u(\mathbf{x}) \sigma n_{1} a\left(t_{0}\right) a(t): t \geq 0\right\}$. It is easily seen that $B_{\mathbf{x}}(o, k a(t) K)=t$. To find $d_{o}(\mathbf{x}, \mathbf{y})$ we also need to find $B_{\mathbf{y}}(o, k a(t) K / K)$. To do this, we need to find $s \in \mathbb{R}$ such that $k a(t) K / K=k^{\prime} n a(s) K / K$ for some $n \in N$ (this $s$ is exactly $B_{\mathbf{y}}(o, k a(t) K / K)$ ).

$$
\begin{aligned}
& u(\mathbf{x}) \sigma n_{1} a\left(t_{0}\right) a(t)=u(\mathbf{y}) \sigma n_{2} n a\left(t_{2}\right) a(s) k " \\
& u(\mathbf{x}-\mathbf{y}) \sigma u\left(\mathbf{x}_{1}\right) a\left(t+t_{0}\right)=\sigma u(\mathbf{z}) a\left(s+t_{2}\right) k,
\end{aligned}
$$

for some $k " \in K$. Here $u\left(\mathbf{x}_{1}\right)=n_{1}$ and $u(\mathbf{z})=n_{2} n$.

Compare the $N A$-coordinates of the two sides of the above equality, we have

$$
\begin{gathered}
\text { LHS }=\left(\frac{e^{t+t_{0}}}{\left(e^{t+t_{0}}\right)^{2}+\left\|\mathbf{x}_{1}\right\|^{2}},(\mathbf{x}-\mathbf{y})-\frac{\mathbf{x}_{1}}{\left(e^{t+t_{0}}\right)^{2}+\left\|\mathbf{x}_{1}\right\|^{2}}\right) \\
\operatorname{RHS}=\left(\frac{e^{s+t_{2}}}{\left(e^{s+t_{2}}\right)^{2}+\|\mathbf{z}\|^{2}},-\frac{x}{\left(e^{s+t_{2}}\right)^{2}+\|\mathbf{z}\|^{2}}\right)
\end{gathered}
$$

Since $\left\|\mathbf{x}_{1}\right\|$ is uniformly bounded, when $t$ is large enough, we have $\frac{e^{t+t_{0}}}{\left(e^{t+t_{0}}\right)^{2}+\left\|\mathbf{x}_{1}\right\|^{2}}$ is very close to $\frac{1}{e^{t+t_{0}}}$ and $\left.\frac{\mathbf{x}_{1}}{\left(e^{t+t_{0}}\right)^{2}+\left\|\mathbf{x}_{1}\right\|^{2}}\right)$ is very small, which means $\left.(\mathbf{x}-\mathbf{y})-\frac{\mathbf{x}_{1}}{\left(e^{t+t_{0}}\right)^{2}+\left\|\mathbf{x}_{1}\right\|^{2}}\right)$ is very close to $\mathbf{x}-\mathbf{y}$. Therefore we have $\frac{e^{s+t_{2}}}{\left(e^{s+t_{2}}\right)^{2}+\|\mathbf{z}\|^{2}} \asymp \frac{1}{e^{t+t_{0}}}$ and $\frac{\|\mathbf{z}\|}{\left(e^{s+t_{2}}\right)^{2}+\|\mathbf{z}\|^{2}} \asymp\|\mathbf{x}-\mathbf{y}\|$, taking the square sum of the above two estimates we have

$$
\frac{1}{\left(e^{s+t_{2}}\right)^{2}+\|\mathbf{z}\|^{2}} \asymp\left(\frac{1}{e^{t+t_{0}}}\right)^{2}+\|\mathbf{x}-\mathbf{y}\|^{2} \asymp\|\mathbf{x}-\mathbf{y}\|^{2}
$$

given $t$ large enough. Therefore we have

This gives that

$$
e^{s+t} \asymp e^{s+t+t_{0}+t_{2}} \asymp\left(e^{s+t_{2}}\right)^{2}+\|\mathbf{z}\|^{2} \asymp \frac{1}{\|\mathbf{x}-\mathbf{y}\|^{2}}
$$

$$
\exp \left(-\frac{1}{2} \lim _{t \rightarrow \infty}\left(B_{\mathbf{x}}(o, k a(t) K / K)+B_{\mathbf{y}}(o, k a(t) K / K)\right)\right) \asymp\|\mathbf{x}-\mathbf{y}\| .
$$


This completes the first part of the proposition.

To compute $\tilde{h}(\gamma \xi \infty)$, we choose $k \in K$ such that $\gamma^{-1} k a(t) \rightarrow \xi \infty$ as $t \rightarrow \infty$, and $k^{\prime} \in K$ such that $k^{\prime} a(s) \rightarrow \xi \infty$ as $s \rightarrow \infty$. Obviously, $k^{\prime}$ corresponding to the vector $v_{\xi} \in T_{o}^{1} \mathbb{H}^{n}$ pointing to $\xi \infty$, this means that $k^{\prime}$ can only be chosen within a fixed finite subset of $K$, depending only on the subgroup $\Gamma$. And then there exists some $t$ such that $\gamma^{-1} k a(t)=\xi n \xi^{-1} k^{\prime}$, this $t$ will be $\log \tilde{h}(\gamma \xi \infty)$ from the properties of Busemann function (note that $\gamma^{-1} k a(t)=g_{t}\left(\gamma^{-1} k\right)$ ). Now, $\gamma^{-1} k a(t) \rightarrow \xi \infty$ means that $\gamma^{-1} k \in \xi P$ which implies $k \in \gamma \xi P$. For the same reason, $k^{\prime} \in \xi P$ which means $\xi^{-1} k^{\prime} \in P$.

Let $\gamma \xi=u\left(\mathbf{x}_{1}\right) \sigma u\left(\mathbf{x}_{2}\right) a(r) m$, then from above we have $k=u\left(\mathbf{x}_{1}\right) \sigma n_{k} a\left(t_{k}\right) m_{k}$ for some $n \in N, m_{k} \in M$ and $a\left(t_{k}\right) \in A$. From our assumption, $\mathbf{x}_{1} \in B$, this shows that $n_{k} \in N$ and $a\left(t_{k}\right)$ are both bounded by some fixed compact subsets (depending on $K$ and $B$ ) of $N$ and $A$ respectively, since $k \in K$ and $u\left(\mathbf{x}_{1}\right) \in u(B)$ are both bounded inside some compact subset $K$ and $u(B)$ respectively. In particular, $t_{k} \in[-C, C]$ for some constant $C>0$ depending on $K$ and $B$. Also, from $\xi^{-1} k^{\prime} \in P$ we have $\xi^{-1} k^{\prime}=n^{\prime} a\left(t_{k^{\prime}}\right) m^{\prime}$, it is clear that $\xi^{-1} k^{\prime}$ is contained some fixed finite subset, which implies $t_{k^{\prime}} \in\left[-C^{\prime}, C^{\prime}\right]$ for some absolute constant depending only on $\Gamma$.

Then from $k a(t)=\gamma \xi n \xi^{-1} k^{\prime}$, we have

$$
u\left(\mathbf{x}_{1}\right) \sigma n_{k} a\left(t_{k}\right) m_{k} a(t)=u\left(\mathbf{x}_{1}\right) \sigma u\left(\mathbf{x}_{2}\right) a(r) m n n^{\prime} a\left(t_{k^{\prime}}\right) m^{\prime}
$$

In the Bruhat decomposition $g=n_{1} \sigma n_{2} a m$, compare the $a$-component of the left and right side of the above equation, we can get $t=r+r_{k^{\prime}}-r_{k}$, given that $r_{k}$ and $r_{k^{\prime}}$ are both bounded from above and below, we have that

$$
e^{t} \asymp e^{r} .
$$

Since $e^{t}=\tilde{h}(\gamma \xi \infty)$ and $e^{r}=h(\gamma \xi \infty)$, this proves the second part of the proposition.

Concerning $\tilde{h}(\cdot)$ and $d_{o}(\cdot, \cdot)$, we have the following interesting equality:

Proposition 3.18. For any $\gamma, \gamma_{1}, \gamma_{2} \in \Gamma$ and $\xi_{1}, \xi_{2} \in \Xi$,

$$
d_{o}^{2}\left(\gamma_{1} \xi_{1} \infty, \gamma_{2} \xi_{2} \infty\right) \tilde{h}\left(\gamma_{1} \xi_{1} \infty\right) \tilde{h}\left(\gamma_{2} \xi_{2} \infty\right)=d_{o}^{2}\left(\gamma \gamma_{1} \xi_{1} \infty, \gamma \gamma_{2} \xi_{2} \infty\right) \tilde{h}\left(\gamma \gamma_{1} \xi_{1} \infty\right) \tilde{h}\left(\gamma \gamma_{2} \xi_{2} \infty\right)
$$

Proof. Let $\left\{\xi_{1}(t): t \geq 0\right\}$ be a geodesic ray pointing to $\xi_{1} \infty$, then

$$
\begin{aligned}
& B_{\gamma_{1} \xi_{1} \infty}\left(o, \gamma_{1} o\right)+B_{\gamma_{2} \xi_{2} \infty}\left(o, \gamma_{2} o\right)-B_{\gamma_{1} \xi_{1} \infty}\left(o, \gamma_{1} \xi_{1}(t)\right)-B_{\gamma_{2} \xi_{2} \infty}\left(o, \gamma_{1} \xi_{1}(t)\right) \\
& =\left(B_{\gamma_{1} \xi_{1} \infty}\left(o, \gamma_{1} o\right)-B_{\gamma_{1} \xi_{1} \infty}\left(o, \gamma_{1} \xi_{1}(t)\right)\right)+\left(B_{\gamma_{2} \xi_{2} \infty}\left(o, \gamma_{2} o\right)-B_{\gamma_{2} \xi_{2} \infty}\left(o, \gamma_{1} \xi_{1}(t)\right)\right) \\
& =B_{\gamma_{1} \xi_{1} \infty}\left(\gamma_{1} \xi_{1}(t), \gamma_{1} o\right)+B_{\gamma_{2} \xi_{2} \infty}\left(\gamma_{1} \xi_{1}(t), \gamma_{2} o\right) \\
& \text { (from the basic properties of Busemann function) } \\
& =B_{\gamma \gamma_{1} \xi_{1} \infty}\left(\gamma \gamma_{1} \xi_{1}(t), \gamma \gamma_{1} o\right)+B_{\gamma \gamma_{2} \xi_{2} \infty}\left(\gamma \gamma_{1} \xi_{1}(t), \gamma \gamma_{2} o\right) \\
& \text { (also from basic properties) } \\
& =\left(B_{\gamma \gamma_{1} \xi_{1} \infty}\left(o, \gamma \gamma_{1} o\right)-B_{\gamma \gamma_{1} \xi_{1} \infty}\left(o, \gamma \gamma_{1} \xi_{1}(t)\right)\right)+\left(B_{\gamma \gamma_{2} \xi_{2} \infty}\left(o, \gamma \gamma_{2} o\right)-B_{\gamma \gamma_{2} \xi_{2} \infty}\left(o, \gamma \gamma_{1} \xi_{1}(t)\right)\right) \\
& =B_{\gamma \gamma_{1} \xi_{1} \infty}\left(o, \gamma \gamma_{1} o\right)+B_{\gamma \gamma_{2} \xi_{2} \infty}\left(o, \gamma \gamma_{2} o\right)-B_{\gamma \gamma_{1} \xi_{1} \infty}\left(o, \gamma \gamma_{1} \xi_{1}(t)\right)-B_{\gamma \gamma_{2} \xi_{2} \infty}\left(o, \gamma \gamma_{1} \xi_{1}(t)\right)
\end{aligned}
$$

By taking the limits of the first and the last expressions in the equations above and applying exponential function, we prove the statement.

Corollary 3.19. For any two cusps $\gamma_{1} \xi_{1} \infty$ and $\gamma_{2} \xi_{2} \infty$ inside $B$, we have

$$
\left\|\gamma_{1} \xi_{1} \infty-\gamma_{2} \xi_{2} \infty\right\| \gg \frac{1}{\sqrt{h\left(\gamma_{1} \xi_{1} \infty\right) h\left(\gamma_{2} \xi_{2} \infty\right)}} .
$$

Proof. We at first prove the above inequality for $\tilde{h}(\cdot)$ and $d_{o}(\cdot, \cdot)$.

We at first consider the case $\gamma_{1}=\gamma$ and $\gamma_{2}=$ id. Choose a particular geodesic ray pointing to $\gamma \xi_{1} \infty$, say $\left\{\gamma \xi_{1} a(t): t \geq 0\right\}$, suppose we have $\gamma \xi_{1}=\xi_{2} n_{1} a(-r) k$ in the representation $G=\xi_{2} N A K$, by the Theorem 2.1, we have $r$ is bounded from below by some absolute constant, then we have

$$
\begin{aligned}
& B_{\xi_{2} \infty}\left(o, \gamma \xi_{1} a(t)\right) \\
= & B_{\xi_{2} \infty}\left(o, \xi_{2} n_{1} a(-r) k a(t)\right)
\end{aligned}
$$

Projecting $n_{1} a(-r) k a(t)$ onto $G / K$ and consider the first component of the $N A$-coordinate (put $k=$ $n_{1} \sigma n_{2} a m$ in Bruhat decomposition and do the same calculation as we did in the proof of Proposition 3.17), 
we have that it is equal to $\frac{e^{t-r}}{e^{2 t}+\|\mathbf{y}\|^{2}}$ for some vector $\mathbf{y} \in \mathbb{R}^{n-1}$, it is clear that it is at most $\frac{e^{t-r}}{e^{2 t}}=e^{-t-r}$. Then we have

$$
\xi_{2} n_{1} a(-r) k a(t)=\xi_{2} n^{\prime} a(-r-t-\epsilon(t)) k
$$

for some $\epsilon(t) \geq 0$. This means that $B_{\xi_{2} \infty}\left(o, \gamma \xi_{1} a(t)\right)=-r-t-\epsilon(t)+t_{1}$, where $t_{1}$ is such that $\xi_{2}=k_{2} a\left(t_{2}\right) n_{2}$.

For $B_{\gamma \xi_{1} \infty}\left(o, \gamma \xi_{1} a(t)\right)$, we have that

$$
\begin{aligned}
& B_{\gamma \xi_{1} \infty}\left(o, \gamma \xi_{1} a(t)\right) \\
= & B_{\gamma \xi_{1} \infty}\left(o, \gamma \xi_{1}\right)+B_{\gamma \xi_{1} \infty}\left(\gamma \xi_{1}, \gamma \xi_{1} a(t)\right) \\
= & B_{\gamma \xi_{1} \infty}(o, \gamma o)+B_{\gamma \xi_{1} \infty}\left(\gamma o, \gamma \xi_{1}\right)+t \\
= & B_{\gamma \xi_{1} \infty}(o, \gamma o)+B_{\xi_{1} \infty}\left(o, \xi_{1}\right)+t
\end{aligned}
$$

We denote $B_{\xi_{1} \infty}\left(o, \xi_{1}\right)$ by $C\left(\xi_{1}\right)$, since it is contained in a fixed finite set, it is uniformly bounded.

Thus, we have

$$
\begin{aligned}
& B_{\gamma \xi_{1} \infty}(o, \gamma o)-B_{\xi_{2} \infty}\left(o, \gamma \xi_{1} a(t)\right)-B_{\gamma \xi_{1} \infty}\left(o, \gamma \xi_{1} a(t)\right) \\
= & B_{\gamma \xi_{1} \infty}(o, \gamma o)-B_{\gamma \xi_{1} \infty}(o, \gamma o)-C\left(\xi_{1}\right)-t+t+r+\epsilon(t)-t_{1} \\
= & r+\epsilon(t)-t_{1}-C\left(\xi_{1}\right) \\
\geq & \tilde{C} .
\end{aligned}
$$

for some absolute constant $\tilde{C}$. By letting $t \rightarrow \infty$ and taking the exponential in the above inequality, we have

$$
d_{o}^{2}\left(\gamma \xi_{1} \infty, \xi_{2} \infty\right) \tilde{h}\left(\gamma \xi_{1} \infty\right) \gg 1 \text {. }
$$

This proves the statement since $\tilde{h}\left(\xi_{2} \infty\right)=1$.

Now we consider the general case. For any two cusps $\gamma_{1} \xi_{1} \infty$ and $\gamma_{2} \xi_{2} \infty$, we have

$$
\begin{aligned}
& \tilde{h}\left(\gamma_{1} \xi_{1} \infty\right) \tilde{h}\left(\gamma_{2} \xi_{2} \infty\right) d_{o}^{2}\left(\gamma_{1} \xi_{1} \infty, \gamma_{2} \xi_{2} \infty\right) \\
= & \tilde{h}\left(\gamma_{2}^{-1} \gamma_{1} \xi_{1} \infty\right) \tilde{h}\left(\xi_{2} \infty\right) d_{o}^{2}\left(\gamma_{2}^{-1} \gamma_{1} \xi_{1}, \xi_{2} \infty\right) \\
\gg & 1 .
\end{aligned}
$$

Now applying the facts that $h(\gamma \xi \infty) \asymp \tilde{h}(\gamma \xi \infty)$ and $d_{o}\left(\gamma_{1} \xi_{1} \infty, \gamma_{2} \xi_{2} \infty\right) \asymp\left\|\gamma_{1} \xi_{1} \infty-\gamma_{2} \xi_{2} \infty\right\|$ whenever the cusps are in $B$, we prove the inequality for $h(\cdot)$ and $\|\cdot\|$.

Definition 3.20. For a cusp $\mathfrak{a}=\gamma \xi \infty$, we call another cusp $\mathfrak{b}$ is a successive cusp of $\mathfrak{a}$ if

$$
\mathfrak{b}=\gamma\left(\xi u(\mathbf{n}) \xi^{-1}\right) s \xi^{\prime} \infty
$$

where $\mathbf{n} \in \mathbb{R}^{n-1}, \xi u(\mathbf{n}) \xi^{-1} \in \Gamma \cap \xi N \xi^{-1}:=\Gamma_{\xi}$ (it is a cocompact lattice of $\xi N \xi^{-1}$ ) with the norm $\|\mathbf{n}\|$ large enough, and $s \notin \Gamma_{\xi}$ is chosen from a finite subset $\mathcal{S} \subset \Gamma$ defined as follows: for any fundamental domain $\mathcal{F}$ that shares a common boundary with $\mathcal{F}_{0}$ is of form $s \mathcal{F}_{0}$, there are only finitely many such fundamental domains, we define $\mathcal{S}$ to be the collection of all the possible $s$ 's. It is easily seen that $\Gamma$ is generated by $\mathcal{S}$.

Proposition 3.21. Given a cusp $\mathfrak{a}=\gamma \xi_{1} \infty$ and one of its successive cusp points $\mathfrak{b}=\gamma \xi_{1} u(\mathbf{n}) \xi_{1}^{-1} s \xi_{2} \infty$, the following approximation is true:

And moreover,

$$
h(\mathfrak{b}) \asymp h(\mathfrak{a})\|\mathbf{n}\|^{2}
$$

$$
\|\mathfrak{a}-\mathfrak{b}\| \asymp \frac{1}{\sqrt{h(\mathfrak{a}) h(\mathfrak{b})}}
$$

Proof. Let $\gamma \xi_{1}=u\left(\mathbf{x}_{1}\right) \sigma u\left(\mathbf{y}_{1}\right) a\left(r_{1}\right) m_{1}$ and $\gamma \xi_{1} u(\mathbf{n}) \xi_{1}^{-1} s \xi_{2}=u\left(\mathbf{x}_{2}\right) \sigma u\left(\mathbf{y}_{2}\right) a\left(r_{2}\right) m_{2}$, we take the inverse of both sides, then the left hand side becomes

$$
\left(\xi_{1}^{-1} s \xi_{2}\right)^{-1} u(-\mathbf{n})\left(\gamma \xi_{1}\right)^{-1}=k^{-1}\left(\xi_{1}, \xi_{2}, s\right) u(-\mathbf{n}) m_{1}^{-1} a\left(-r_{1}\right) u\left(-\mathbf{y}_{1}\right) \sigma u\left(-\mathbf{x}_{1}\right)
$$

where $k\left(\xi_{1}, \xi_{2}, s\right)$ denotes $\xi_{1}^{-1} s \xi_{2}$ for $\xi_{1}, \xi_{2} \in \Xi$ and $s \in \mathcal{S}$. we claim that $k\left(\xi_{1}, \xi_{2}, s\right) \notin P$ unless $s=e$ and $\xi_{1}=\xi_{2}$. This is because if this happens then $\xi_{1} \infty=s \xi_{2} \infty$, this means that they represent exact the same cusp.

We denote $k\left(\xi_{1}, \xi_{2}, s\right)=u\left(\mathbf{x}\left(\xi_{1}, \xi_{2}, s\right)\right) \sigma a\left(r\left(\xi_{1}, \xi_{2}, s\right)\right) u\left(\mathbf{y}\left(\xi_{1}, \xi_{2}, s\right)\right) m\left(\xi_{1}, \xi_{2}, s\right)$, since we only have finitely many choices for $\xi_{1}, \xi_{2}$ and $s$, we have $\mathbf{x}\left(\xi_{1}, \xi_{2}, s\right), r\left(\xi_{1}, \xi_{2}, s\right)$ and $\mathbf{y}\left(\xi_{1}, \xi_{2}, s\right)$ are all bounded. This makes the left hand side equal

$$
m^{-1}\left(\xi_{1}, \xi_{2}, s\right) u\left(-\mathbf{y}\left(\xi_{1}, \xi_{2}, s\right)\right) a\left(-r\left(\xi_{1}, \xi_{2}, s\right)\right) \sigma u\left(-\mathbf{x}\left(\xi_{1}, \xi_{2}, s\right)\right) u(-\mathbf{n}) m_{1}^{-1} a\left(-r_{1}\right) u\left(-\mathbf{y}_{1}\right) \sigma u\left(-\mathbf{x}_{1}\right)
$$


The right hand side is equal to

$$
m_{2}^{-1} a\left(-r_{2}\right) u\left(-\mathbf{y}_{2}\right) \sigma u\left(-\mathbf{x}_{2}\right)
$$

Now we consider their $N A$-coordinates on $G / K$, the right hand side has coordinate

$$
\left(\frac{e^{-r_{2}}}{1+\left\|\mathbf{x}_{2}\right\|^{2}}, e^{-r_{2}} m_{2}^{-1}\left(\frac{\mathbf{x}_{2}}{1+\left\|\mathbf{x}_{2}\right\|^{2}}-\mathbf{y}_{2}\right)\right) \text {. }
$$

The left hand side is equal to:

$$
\left(e^{-r\left(\xi_{1}, \xi_{2}, s\right)} \frac{A}{A^{2}+\|\mathbf{B}\|^{2}}, m^{-1}\left(\xi_{1}, \xi_{2}, s\right)\left(-\mathbf{y}\left(\xi_{1}, \xi_{2}, s\right)+e^{-r\left(\xi_{1}, \xi_{2}, s\right)} \frac{\mathbf{B}}{A^{2}+\|\mathbf{B}\|^{2}}\right)\right),
$$

where $A=\frac{e^{-r_{1}}}{1+\left\|\mathbf{x}_{1}\right\|^{2}}$ and $\mathbf{B}=m_{1}^{-1}\left(\frac{\mathbf{x}_{1}}{1+\left\|\mathbf{x}_{1}\right\|^{2}}-\mathbf{y}_{1}\right)-\mathbf{n}-\mathbf{x}\left(\xi_{1}, \xi_{2}, s\right)$, since we assume that $\|\mathbf{n}\|$ is large enough, we have $\|\mathbf{B}\| \asymp\|\mathbf{n}\|$, and since $\left\|\mathbf{x}_{1}\right\| \ll 1$ and $e^{r\left(\xi_{1}, \xi_{2}, s\right)} \asymp 1$, we have that

$$
e^{-r\left(\xi_{1}, \xi_{2}, s\right)} \frac{A}{A^{2}+\|\mathbf{B}\|^{2}} \asymp \frac{e^{-r_{1}}}{\|\mathbf{n}\|^{2}}
$$

and the first coordinate of right hand side $\frac{e^{-r_{2}}}{1+\left\|\mathbf{x}_{2}\right\|^{2}} \asymp e^{-r_{2}}$ since $\left\|\mathbf{x}_{1}\right\| \ll 1$. Therefore, we have

$$
e^{r_{2}} \asymp e^{r_{1}}\|\mathbf{n}\|^{2} \text {. }
$$

This proves the first part of the proposition.

Moreover, by comparing the second component of the coordinate, we have

$$
e^{-r_{2}} m_{2}^{-1}\left(\frac{\mathbf{x}_{2}}{1+\left\|\mathbf{x}_{2}\right\|^{2}}-\mathbf{y}_{2}\right)=m^{-1}\left(\xi_{1}, \xi_{2}, s\right)\left(-\mathbf{y}\left(\xi_{1}, \xi_{2}, s\right)+e^{-r\left(\xi_{1}, \xi_{2}, s\right)} \frac{\mathbf{B}}{A^{2}+\|\mathbf{B}\|^{2}}\right) .
$$

The right hand side has uniformly bounded norm, this shows that the left hand side is also uniformly bounded. Thus,

$$
\left\|\frac{\mathbf{x}_{2}}{1+\left\|\mathbf{x}_{2}\right\|^{2}}-\mathbf{y}_{2}\right\| \ll e^{r_{2}} .
$$

It is easily seen that the norm of $\frac{\mathbf{x}_{2}}{1+\left\|\mathbf{x}_{2}\right\|^{2}}$ is uniformly bounded. This shows that

$$
\left\|\mathbf{y}_{2}\right\| \ll e^{r_{2}}=h(\mathfrak{b}) \text {. }
$$

Remark: here we could not assume that $\left\|\mathbf{y}_{2}\right\| \ll h(\mathfrak{b})$ (as we mentioned in the remark after Definition 3.1), since this will change $\gamma \xi_{1} u(\mathbf{n}) \xi_{1}^{-1} s \xi_{2}$ to $\gamma \xi_{1} u(\mathbf{n}) \xi_{1}^{-1} s \xi_{2} u\left(\mathbf{n}^{\prime}\right)$ for some $\mathbf{n}^{\prime} \in \mathbb{R}^{n-1}$, then the following equality will not hold anymore:

$$
\left.u\left(\mathbf{x}_{1}\right) \sigma u\left(\mathbf{y}_{1}\right) a\left(r_{1}\right) m_{1} u(\mathbf{n}) \xi_{1}^{-1} s \xi_{2}=u\left(\mathbf{x}_{2}\right) \sigma u\left(\mathbf{y}_{2}\right) a\left(r_{2}\right) m_{2} \cdot\right)
$$

Now we start with

$$
\gamma \xi_{1} u(\mathbf{n}) \xi_{1}^{-1} s \xi_{2}=u\left(\mathbf{x}_{1}\right) \sigma u\left(\mathbf{y}_{1}\right) a\left(r_{1}\right) m_{1} u(\mathbf{n}) k\left(\xi_{1}, \xi_{2}, s\right)
$$

on the other hand,

$$
\gamma \xi_{1} u(\mathbf{n}) \xi_{1}^{-1} s \xi_{2}=u\left(\mathbf{x}_{2}\right) \sigma u\left(\mathbf{y}_{2}\right) a\left(r_{2}\right) m_{2}
$$

Suppose the $N A$-coordinate of $k\left(\xi_{1}, \xi_{2}, s\right)$ is $\left(e^{r}(\xi, s), \mathbf{z}(\xi, s)\right)$, then the $N A$-coordinate of $\gamma \xi_{1} u(\mathbf{n}) \xi_{1}^{-1} s \xi_{2}$ is the following, by plugging in the first equation:

$$
\left(\frac{C}{C^{2}+\|\mathbf{D}\|^{2}}, \mathbf{x}_{1}-\frac{\mathbf{D}}{C^{2}+\|\mathbf{D}\|^{2}}\right),
$$

where $C=e^{r_{1}+r(\xi, s)} \asymp h(\mathbf{a}), \mathbf{D}=\mathbf{y}_{1}+e^{r_{1}} m_{1}(\mathbf{n}+\mathbf{z}(\xi, s))$, for $\|\mathbf{n}\|$ large enough, $\|\mathbf{D}\| \asymp h(\mathfrak{a})\|\mathbf{n}\|$. So

$$
\left\|\frac{\mathbf{D}}{C^{2}+\|\mathbf{D}\|^{2}}\right\| \asymp \frac{1}{h(\mathfrak{a})\|\mathbf{n}\|} .
$$

By plugging the second equation into the $N A$-coordinate, we have

$$
\left(\frac{e^{r_{2}}}{e^{2 r_{2}}+\left\|\mathbf{y}_{2}\right\|^{2}}, \mathbf{x}_{2}-\frac{\mathbf{y}_{2}}{e^{2 r_{2}}+\left\|\mathbf{y}_{2}\right\|^{2}}\right) \text {. }
$$


We have proved that $\left\|\mathbf{y}_{2}\right\| \ll e^{r_{2}}=h(\mathfrak{b})$, so

$$
\left\|\frac{\mathbf{y}_{2}}{e^{2 r_{2}}+\left\|\mathbf{y}_{2}\right\|^{2}}\right\| \ll \frac{1}{h(\mathfrak{b})} \ll \frac{1}{h(\mathfrak{a})\|\mathbf{n}\|} .
$$

By comparing the second component of their $N A$-coordinates, we get

therefore

$$
\mathbf{x}_{1}-\frac{\mathbf{D}}{C^{2}+\|\mathbf{D}\|^{2}}=\mathbf{x}_{2}-\frac{\mathbf{y}_{2}}{e^{2 r_{2}}+\left\|\mathbf{y}_{2}\right\|^{2}}
$$

$$
\mathbf{x}_{1}-\mathbf{x}_{2}=\frac{\mathbf{D}}{C^{2}+\|\mathbf{D}\|^{2}}-\frac{\mathbf{y}_{2}}{e^{2 r_{2}}+\left\|\mathbf{y}_{2}\right\|^{2}}
$$

our above argument shows that the right hand side has norm $\asymp \frac{1}{h(\mathfrak{a})\|\mathbf{n}\|} \asymp \frac{1}{\sqrt{h(\mathfrak{a}) h(\mathfrak{b})}}$, this shows that

$$
\left\|\mathbf{x}_{1}-\mathbf{x}_{2}\right\| \asymp \frac{1}{\sqrt{h(\mathfrak{a}) h(\mathfrak{b})}} .
$$

This proves the second part of the proposition.

Corollary 3.22. There exist constants $\epsilon>0$ and $C>0$ such that for a cusp $\mathfrak{a}$ and one of its successive cusp points $\mathfrak{b}$, then for any

$$
\mathbf{x} \in B\left(\mathfrak{b}, \frac{\epsilon}{h(\mathfrak{b})}\right)=\left\{\mathbf{x} \in \mathbb{R}^{n-1}:\|\mathbf{x}-\mathfrak{b}\|<\frac{\epsilon}{h(\mathfrak{b})}\right\},
$$

$\mathcal{G}_{\mathbf{x}}$ enters both $\mathfrak{a}$ and $\mathfrak{b}$, moreover, from leaving $\mathfrak{a}$ to entering $\mathfrak{b}$, it spends at most time $C$.

Proof. For $\epsilon>0$ small enough, then if

the following is also true:

$$
\|\mathbf{x}-\mathfrak{b}\| \leq \frac{\epsilon}{h(\mathfrak{b})}
$$

$$
\|\mathbf{x}-\mathfrak{a}\| \leq \frac{c}{h(\mathfrak{a})},
$$

since $\|\mathfrak{b}-\mathfrak{a}\| \asymp \frac{1}{\sqrt{h(\mathfrak{a}) h(\mathfrak{b})}} \ll \frac{1}{h(\mathfrak{a})}$ (see Proposition 3.21). Therefore $\mathcal{G}_{\mathbf{x}}$ enters both $\mathfrak{a}$ and $\mathfrak{b}$.

The time $t_{2}(\mathfrak{a})$ when $\mathcal{G}_{\mathbf{x}}$ leaves $\mathfrak{a}$ satisfies:

$$
e^{t_{2}(\mathfrak{a})} \asymp \frac{1}{h(\mathfrak{a})\|\mathbf{x}-\mathfrak{a}\|^{2}} \asymp h(\mathfrak{b}),
$$

and the time $t_{1}(\mathfrak{b})$ when $\mathcal{G}_{\mathbf{x}}$ enters $\mathfrak{b}$ satisfies:

$$
e^{t_{1}(\mathfrak{b})} \asymp h(\mathfrak{b}) .
$$

Therefore $e^{t_{2}(\mathfrak{a})} \asymp e^{t_{1}(\mathfrak{b})}$, which is equivalent to our conclusion.

For $\mathbf{x} \in \mathbb{R}^{n-1}$, if we consider the rough spectrum of $\mathbf{x}$, say $\left\{\mathfrak{a}_{i}: i \in \mathbb{N}\right\}$, we have the following result:

Proposition 3.23. Let $\mathbf{x} \in \mathbb{R}^{n-1}$, we denote the rough spectrum of $\mathbf{x}$ by $\left\{\mathfrak{a}_{i}: i \in \mathbb{N}\right\}$, then for each $i \in \mathbb{N}$,

$$
\left\|\mathbf{x}-\mathfrak{a}_{i}\right\| \asymp \frac{1}{\sqrt{h\left(\mathfrak{a}_{i}\right) h\left(\mathfrak{a}_{i+1}\right)}} .
$$

And moreover, for each $i \in \mathbb{N}, \mathfrak{a}_{i+1}$ is a successive cusp of $\mathfrak{a}_{i}$

Proof. By Corollary 3.13, the time $t$ when $\mathcal{G}_{\mathbf{x}}$ roughly leaves $\mathfrak{a}_{i}$ and roughly enters $\mathfrak{a}_{i+1}$ satisfies

and

$$
e^{t} \asymp \frac{1}{h\left(\mathfrak{a}_{i}\right)\left\|\mathbf{x}-\mathfrak{a}_{i}\right\|^{2}}
$$

this implies that

$$
e^{t} \asymp h\left(\mathfrak{a}_{i+1}\right),
$$

$$
\left\|\mathbf{x}-\mathfrak{a}_{i+1}\right\| \asymp \frac{1}{\sqrt{h\left(\mathfrak{a}_{i}\right) h\left(\mathfrak{a}_{i+1}\right)}} .
$$


The proof of the second statement goes as follows: suppose when $\mathcal{G}_{\mathbf{x}}$ roughly enters $\mathfrak{a}_{i}$, it is in the fundamental domain $\gamma \mathcal{F}_{0}$, then $\mathfrak{a}_{i}=\gamma \xi \infty$, where $\xi \infty$ is some cusp point of $\mathcal{F}_{0}$. When $\mathcal{G}_{\mathbf{x}}$ leaves $\mathfrak{a}_{i}$, the fundamental domain it leaves must contain $\gamma \xi \infty$ as a cusp point, therefore the fundemantal domain is of form $\gamma \xi u(\mathbf{n}) \xi^{-1} \mathcal{F}_{0}$ where $\xi u(\mathbf{n}) \xi^{-1} \in \Gamma_{\xi}$. Then when $\mathcal{G}_{\mathbf{x}}$ roughly enters $\mathfrak{a}_{i+1}$, the fundamental domain it enters must be adjacent to $\gamma \xi u(\mathbf{n}) \xi^{-1}$, this implies the cusp point $\mathfrak{a}_{i+1}$ must be of form $\gamma \xi u(\mathbf{n}) \xi^{-1} s \xi^{\prime} \infty$ for some $s \in \mathcal{S}$ and some other cusp $\xi^{\prime} \infty$ of $\mathcal{F}_{0}$. This completes the proof.

\section{Counting CUSP POINTS In A GIVEN REgion}

In this section we will prove the counting result on cusp points inside a given region with heights in a given range, as we mentioned in the introduction (see Problem 1.2).

Theorem 4.1. There exist constants $A_{3}>0, A_{1}<1<A_{2}, T>0, h>0$ and $\Upsilon>0$ such that for any $t \geq T$, and for any $\gamma \in \Gamma$ satisfying the cusp $\mathfrak{a}=\gamma \infty \in B$, and $h(\mathfrak{a}) \geq h$, we have the number of cusp points $\mathfrak{b}$ of form $\gamma^{\prime} \infty$ such that $h(\mathfrak{b}) \in\left[A_{1} e^{t} h(\mathfrak{a}), A_{2} e^{t} h(\mathfrak{a})\right]$ and $\mathfrak{b} \in B\left(\gamma \infty, \frac{A_{3}}{h(\mathfrak{a})}\right)$ is at least $\Upsilon e^{(n-1) t}$.

Remark 4.2. The basic argument of the proof is based on the idea in the thesis of Margulis on counting closed geodesics in compact Riemannian manifold of negative curvature. The basic tool is the mixing of geodesic flow on hyperbolic space of finite volume.

The basic idea of the proof goes as follows: given $\epsilon>0$, we take a neighborhood $\Omega$ of id in $G$ of form $\mathcal{N}_{\epsilon} \mathcal{A}_{\epsilon} \mathcal{U}_{\epsilon}^{-} M$, where $\mathcal{N}_{\epsilon}, \mathcal{A}_{\epsilon}$ and $\mathcal{U}_{\epsilon}^{-}$are $\epsilon$-neighborhoods of id in $N, A$ and $U^{-}$, respectively, such that it maps to $\Gamma \backslash G$ injectively under the natural projection:

$$
\pi: G \rightarrow \Gamma \backslash G
$$

From the mixing property of geodesic flow on $\mathrm{T}^{1}\left(\Gamma \backslash \mathbb{H}^{n}\right)$ with respect to the Lebesgue measure $\mu_{G}$, we have when $t>0$ large enough,

$$
\mu_{G}(\Gamma \Omega a(t) \cap \Gamma \Omega) \geq \frac{9}{10}\left(\mu_{G}(\Gamma \Omega)\right)^{2} .
$$

Unfolding the above intersection to $\mathrm{T}^{1}\left(\mathbb{H}^{n}\right)$, the left hand side is equal to

$$
\sum_{\gamma^{\prime} \in \Gamma} \mu\left(\gamma \Omega a(t) \cap \gamma^{\prime} \Omega\right)
$$

where $\mu$ denotes the $G$-invariant Lebesgue measure on $\mathrm{T}^{1}\left(\mathbb{H}^{n}\right)$. By a result proved in [10],

$$
\mu\left(\gamma \Omega a(t) \cap \gamma^{\prime} \Omega\right) \leq C(\Omega) e^{-(n-1) t},
$$

where $C(\Omega)>0$ is a constant depending on $\Omega$. Then there exists a constant $\Upsilon>0$ such that there are at least $\Upsilon e^{(n-1) t} \gamma^{\prime} \in \Gamma$ such that

$$
\gamma \Omega a(t) \cap \gamma^{\prime} \Omega \neq \emptyset .
$$

Each such $\gamma^{\prime}$ will be proved to satisfy the properties described in Theorem 4.1 which finishes the proof.

We start with proving the following lemma:

Lemma 4.3. There exist constants $\epsilon>0, A_{3}>0, A_{2}>1>A_{3}$, such that: let $\Omega \subset G$ denote the $\epsilon$ neighborhood of id of form $\mathcal{N}_{\epsilon} \mathcal{A}_{\epsilon} \mathcal{U}_{\epsilon}^{-} M$, given any $\gamma \in \Gamma$ such that $\mathfrak{a}=\gamma \infty \in B$ and $h(\mathfrak{a})$ is large enough, $t>0$ large enough, any $\gamma^{\prime}$ such that

$$
\gamma \Omega a(t) \cap \gamma^{\prime} \Omega \neq \emptyset
$$

satisfies the following:

- $\left\|\gamma^{\prime} \infty-\mathfrak{a}\right\| \leq \frac{A_{3}}{h(\mathfrak{a})}$.

- $h\left(\gamma^{\prime} \infty\right) \in\left[A_{1} e^{t} h(\mathfrak{a}), A_{2} e^{t} h(\mathfrak{a})\right]$.

Proof. By the definition of $\gamma^{\prime}$, there exist $w_{1}, w_{2} \in \Omega$ such that

$$
\gamma w_{1} a(t)=\gamma^{\prime} w_{2} \text {. }
$$

We use $\tilde{h}(\gamma \infty)$ and $\tilde{h}\left(\gamma^{\prime} \infty\right)$. Suppose $\gamma=k a(r) u(\mathbf{x})$, then from the definition of $\tilde{h}(\cdot), \tilde{h}(\gamma \infty)=e^{r}$. Since $\Gamma_{\infty}=\Gamma \cap N$ is a lattice of $N$, we could replace $\gamma$ by $\gamma u(\mathbf{n})$ for appropriate $u(\mathbf{n}) \in \Gamma_{\infty}$ to make $\|\mathbf{x}\| \asymp 1$. 
Suppose $w_{1}=u\left(\mathbf{x}_{1}\right) a\left(\epsilon_{1}\right) u^{-}\left(\mathbf{y}_{1}\right) m_{1}, w_{2}=u\left(\mathbf{x}_{2}\right) a\left(\epsilon_{2}\right) u^{-}\left(\mathbf{y}_{2}\right) m_{2}$, where $\left\|\epsilon_{i}\right\|<\epsilon,\left\|\mathbf{x}_{i}\right\| \leq \epsilon$ and $\left\|\mathbf{y}_{i}\right\| \leq \epsilon$ for $i=1,2$. Then

$$
\gamma u\left(\mathbf{x}_{1}\right) a\left(\epsilon_{1}\right) u^{-}\left(\mathbf{y}_{1}\right) m_{1} a(t) m_{2}^{-1} u^{-}\left(-\mathbf{y}_{2}\right)=\gamma^{\prime} u\left(\mathbf{x}_{2}\right) a\left(\epsilon_{2}\right) .
$$

Plugging in $\gamma=k a(r) u(\mathbf{x})$, the left hand side is equal to

$$
\text { LHS }=k^{\prime} a\left(r+\epsilon_{1}\right) u\left(e^{-\epsilon_{1}} m\left(\mathbf{x}+\mathbf{x}_{1}\right)\right) u^{-}\left(m \mathbf{y}_{1}-e^{-t} \mathbf{y}_{2}\right) a(t),
$$

where $m=m_{2} m_{1}^{-1}$, and $k^{\prime}=k m^{-1} \in K$. Denote $\tilde{r}=r+\epsilon_{1}, e^{-\epsilon_{1}} m\left(\mathbf{x}+\mathbf{x}_{1}\right)=\tilde{\mathbf{x}}$, and denote $\tilde{\mathbf{y}}=m \mathbf{y}_{1}-e^{-t} \mathbf{y}_{2}$, then $\tilde{r}$ is close to $r,\|\tilde{\mathbf{x}}\| \asymp 1$ and $\|\tilde{\mathbf{y}}\|$ is very small, then

$$
\text { LHS }=k^{\prime} a(\tilde{r}) u(\tilde{\mathbf{x}}) u^{-}(\tilde{\mathbf{y}}) a(t),
$$

we want to write $k^{\prime} a(\tilde{r}) u(\tilde{\mathbf{x}}) u^{-}(\tilde{\mathbf{y}})$ in terms of $K A N$-decomposition. To do this, we consider its inverse

$$
u^{-}(-\tilde{\mathbf{y}}) u(-\tilde{\mathbf{x}}) a(-\tilde{r}) k^{-1}
$$

and calculate its $N A$-coordinate: we at first write $u^{-}(-\tilde{\mathbf{y}})=\sigma u(-\tilde{\mathbf{y}}) \sigma$, then direct computation shows that its $N A$-coordinate is the following:

$$
\sigma\left(\frac{e^{-\tilde{r}}}{e^{-2 \tilde{r}}+\|\tilde{\mathbf{x}}\|^{2}}, \frac{\tilde{\mathbf{x}}}{e^{-2 \tilde{r}}+\|\tilde{\mathbf{x}}\|^{2}}-\tilde{\mathbf{y}}\right)
$$

we denote

$$
\eta=\frac{e^{-\tilde{r}}}{e^{-2 \tilde{r}}+\|\tilde{\mathbf{x}}\|^{2}}
$$

and

$$
\mathbf{Z}=\frac{\tilde{\mathbf{x}}}{e^{-2 \tilde{r}}+\|\tilde{\mathbf{x}}\|^{2}}-\tilde{\mathbf{y}}
$$

it is easily seen that $\eta \asymp e^{-\tilde{r}}$, and $\|\mathbf{Z}\|$ is close to $\|\tilde{x}\|^{-1} \asymp 1$. Then after applying the action of $\sigma$ on $(\eta, \mathbf{Z})$, we get

the $A$-component is

$$
\left(\frac{\eta}{\eta^{2}+\|\mathbf{Z}\|^{2}},-\frac{\mathbf{Z}}{\eta^{2}+\|\mathbf{Z}\|^{2}}\right)
$$

and the $N$-component is

$$
\frac{\eta}{\eta^{2}+\|\mathbf{Z}\|^{2}} \asymp \frac{\eta}{\|\mathbf{Z}\|^{2}} \asymp e^{-r}
$$

$$
-\frac{\mathbf{Z}}{\eta^{2}+\|\mathbf{Z}\|^{2}}:=-\tilde{\mathbf{Z}}
$$

has norm $\|\tilde{\mathbf{Z}}\| \asymp \frac{1}{\|\mathbf{Z}\|} \asymp 1$. This shows that

$$
k^{\prime} a(\tilde{r}) u(\tilde{\mathbf{x}}) u^{-}(\tilde{\mathbf{y}})=k^{\prime \prime} a\left(r^{\prime}\right) u(\tilde{\mathbf{Z}}),
$$

where $\left|r-r^{\prime}\right|$ is bounded by a constant. Then

$$
k^{\prime} a(\tilde{r}) u(\tilde{\mathbf{x}}) u^{-}(\tilde{\mathbf{y}}) a(t)=k^{\prime \prime} a\left(r^{\prime}+t\right) u\left(e^{-t} \tilde{\mathbf{Z}}\right),
$$

therefore

This easily implies

$$
\gamma^{\prime} u\left(\mathbf{x}_{2}\right) a\left(\epsilon_{2}\right)=k^{\prime \prime} a\left(r^{\prime}+t\right) u\left(e^{-t} \tilde{\mathbf{Z}}\right) .
$$

this shows that $\tilde{h}\left(\gamma^{\prime} \infty\right)=e^{r^{\prime}+t-\epsilon_{2}} \asymp e^{t} \tilde{h}(\gamma \infty)$.

$$
\gamma^{\prime}=k^{\prime \prime} a\left(r^{\prime}+t-\epsilon_{2}\right) u\left(e^{\epsilon_{2}-t} \tilde{\mathbf{Z}}-\mathbf{x}_{2}\right),
$$

Next we want to prove that

$$
\left\|\gamma^{\prime} \infty-\gamma \infty\right\| \leq \frac{A_{3}}{h(\mathfrak{a})} .
$$

To do this, we firstly consider the Gromov metric $d_{o}(\cdot, \cdot)$, and then make use of the fact that

$$
d_{o}\left(\xi_{1}, \xi_{2}\right) \asymp\left\|\xi_{1}-\xi_{2}\right\|
$$

when $\xi_{1}, \xi_{2} \in B$.

For a noncusp point $\gamma w_{1} \infty$ with $w_{1} \in \Omega$, we could define the $\tilde{h}(\cdot)$ height of $\gamma w_{1} \infty$ similarly

$$
\tilde{h}\left(\gamma w_{1} \infty\right)=\exp \left(B_{w_{1} \infty}\left(\gamma^{-1} o, o\right)\right) \text {. }
$$


Then the above argument shows that

$$
\tilde{h}\left(\gamma w_{1} \infty\right) \asymp \tilde{h}(\gamma \infty) .
$$

Then Proposition 3.18 tells that

$$
\begin{aligned}
& d_{o}^{2}\left(\gamma \infty, \gamma w_{1} \infty\right) \tilde{h}(\gamma \infty) \tilde{h}\left(\gamma w_{1} \infty\right) \\
= & d_{o}^{2}\left(\infty, w_{1} \infty\right) \tilde{h}(\infty) \tilde{h}\left(w_{1} \infty\right) \\
= & d_{o}^{2}\left(\infty, w_{1} \infty\right) \\
\ll & 1
\end{aligned}
$$

The last inequality above follows from the basic properties of Gromov metric. So we have

$$
d_{o}\left(\gamma \infty, \gamma w_{1} \infty\right) \ll \frac{1}{\tilde{h}(\gamma \infty)} .
$$

Similarly, we have

$$
d_{o}\left(\gamma^{\prime} \infty, \gamma^{\prime} w_{2} \infty\right) \ll \frac{1}{\tilde{h}\left(\gamma^{\prime} \infty\right)} .
$$

Since $\gamma^{\prime} w_{2} \infty=\gamma w_{1} a(t) \infty=\gamma w_{1} \infty$, and since $\tilde{h}\left(\gamma^{\prime} \infty\right) \asymp e^{t} \tilde{h}(\gamma \infty)$, the following holds:

$$
d_{o}\left(\gamma \infty, \gamma^{\prime} \infty\right) \ll \frac{1}{\tilde{h}(\gamma \infty)} .
$$

This completes the proof because when $\gamma \infty, \gamma^{\prime} \infty \in B$,

and

$$
\begin{aligned}
\left\|\gamma \infty-\gamma^{\prime} \infty\right\| & \asymp d_{o}\left(\gamma \infty, \gamma^{\prime} \infty\right), \\
\tilde{h}(\gamma \infty) & \asymp h(\gamma \infty),
\end{aligned}
$$

$$
\tilde{h}\left(\gamma^{\prime} \infty\right) \asymp h\left(\gamma^{\prime} \infty\right)
$$

We will need the following result of Gorodnik and Shah:

Proposition 4.4. (See [10, Proposition 3.2]) Let $G$ be a real algebraic group, and $\sigma$ is an involution of $G$. Let $A=\{a(t)\}$ be a one parameter subgroup of $G$, such that $\sigma(a(t))=a(-t)$. Let

$$
\begin{array}{ll}
H=\{g \in G: \sigma(g)=g\} & U^{+}=\{g \in G: a(-t) g a(t) \rightarrow e \text { as } t \rightarrow \infty\} \\
U^{-}=\{g \in G: a(t) g a(-t) \rightarrow e \text { as } t \rightarrow \infty\} & Z=Z_{G}(A)
\end{array}
$$

Then there exist constant $c$ and $t_{0}$ such that for neighborhoods $H_{r_{1}} \subset H, Z_{r_{2}} \subset Z$ and $U_{r_{3}}^{+}$of $e$ in $H, Z$ and $U^{+}$, respectively, small enough, $t>t_{0}$ and any $g \in G$, we have

$$
\mu_{G}\left(g U_{r_{3}}^{+} Z_{r_{2}} H_{r_{1}} \cap U_{r_{3}}^{+} Z_{r_{2}} H_{r_{1}} a(-t)\right) \leq c e^{-\lambda t} \mu_{U^{+}}\left(U_{r_{3}}^{+}\right)^{2}
$$

where $\mu_{U^{+}}$denotes the Haar measure on $U^{+}$, and $\lambda$ is the sum of the eigenvalues of $\operatorname{Ad}(a(1))$ which are greater than 1.

Remark 4.5. In our case, we take $G=\mathrm{SO}(n, 1)$ and $\sigma$ be the weyl element, then we have $H=K \cong \mathrm{SO}(n)$ and $\lambda=n-1$. We could choose $r_{1}, r_{2}$ and $r_{3}$ appropriately such that $\Omega \subset U_{r_{3}}^{+} Z_{r_{2}} H_{r_{1}}$. Then the above proposition tells that

$$
\mu\left(\gamma \Omega a(t) \cap \gamma^{\prime} \Omega\right) \leq C(\Omega) e^{-(n-1) t},
$$

for some constant $C(\Omega)$ depending on $\Omega$.

proof of Theorem 4.1. As we mentioned before, the diagonal flow $A=\{a(t): t \in \mathbb{R}\}$ is mixing on $\Gamma \backslash G$ with respect to the finite $G$-invariant measure $\mu_{G}$. So there exists a constant $T>0$ such that for $t>T$,

$$
\mu_{G}(\Gamma \Omega a(t) \cap \Gamma \Omega) \geq \frac{9}{10}\left(\mu_{G}(\Gamma \Omega)\right)^{2} .
$$

Let $\gamma \in \Gamma$ be as above, then by unfolding $\Gamma \Omega a(t) \cap \Gamma \Omega$ to $G$, we have

$$
\mu_{G}(\Gamma \Omega a(t) \cap \Gamma \Omega)=\sum_{\gamma^{\prime} \in \Gamma} \mu\left(\gamma \Omega a(t) \cap \gamma^{\prime} \Omega\right) .
$$


By $(*)$, we get $\left|\left\{\gamma^{\prime} \in \Gamma: \gamma \Omega a(t) \cap \gamma^{\prime} \Omega \neq \emptyset\right\}\right| \geq \Upsilon e^{(n-1) t}$ for some constant $\Upsilon>0$. Here $|\cdot|$ denotes the cardinality of a set. Then applying Lemma 4.3, we finish the proof of Theorem 4.1 because any such $\gamma^{\prime}$ satisfies:

- $\left\|\gamma^{\prime} \infty-\mathfrak{a}\right\| \leq \frac{A_{3}}{h(\mathfrak{a})}$,

- $h\left(\gamma^{\prime} \infty\right) \in\left[A_{1} e^{t} h(\mathfrak{a}), A_{2} e^{t} h(\mathfrak{a})\right]$,

for some constants $0<A_{1}<1<A_{2}$ and $A_{3}>0$.

\section{HAUSdORFF Dimension OF Divergent TRAJECTORIES UNDER DIAGONAL GEODESIC FlOW}

In this section, we will compute the Hausdorff dimension of $\mathfrak{D}_{k}$. In the first subsection, we will give the lower bound of the Hausdorff dimension, and the second subsection will be devoted to the proof of upper bound of the Hausdorff dimension.

Given $\mathcal{V}_{k}=\left(\mathbf{x}_{1}, \mathbf{x}_{2}, \ldots, \mathbf{x}_{k}\right) \in \mathbb{R}^{n-1}$, we denote by $\mathcal{G}\left(\mathcal{V}_{k}\right)$ the diagonal geodesic ray in $\mathcal{M}_{k}$ :

$$
\mathcal{G}\left(\mathcal{V}_{k}\right):=\left\{\mathcal{G}\left(\mathcal{V}_{k}, t\right)=\left(\Gamma_{1} u\left(\mathbf{x}_{1}\right) \sigma a(t), \ldots, \Gamma_{k} u\left(\mathbf{x}_{k}\right) \sigma a(t)\right): t \geq 0\right\} .
$$

We define the function $W\left(\mathcal{V}_{k}, t\right)$ as follows:

$$
W\left(\mathcal{V}_{k}, t\right)=\max _{1 \leq i \leq k}\left\{W_{i}\left(\mathbf{x}_{i}, t\right)\right\}
$$

where $W_{i}(\mathbf{x}, t)$ denotes the function $W(\mathbf{x}, t)$ defined in Definition 3.7 with respect to the space $\Gamma_{i} \backslash \mathbb{H}^{n}$. Then $\mathcal{G}\left(\mathcal{V}_{k}, t\right)$ diverges as $t \rightarrow \infty$ if and only if $W\left(\mathcal{V}_{k}, t\right) \rightarrow \infty$ as $t \rightarrow \infty$.

Because we are interested in divergent trajectories, we may assume that $W\left(\mathcal{V}_{k}, t\right)$ remains large for all $t>0$ large enough.

Suppose at some point $t$,

$$
W\left(\mathcal{V}_{k}, t\right)=W_{i}\left(\mathbf{x}_{i}, t\right)
$$

then there exists a maximal interval $I$ such that for all $s \in I$,

$$
W\left(\mathcal{V}_{k}, s\right)=W_{i}\left(\mathbf{x}_{i}, s\right) .
$$

Then from the properties of the function $W_{i}\left(\mathbf{x}_{i}, t\right)$,

$$
W_{i}\left(\mathbf{x}_{i}, t\right)=\frac{1}{h(\mathfrak{a})\left(e^{-t}+e^{t}\left\|\mathbf{x}_{i}-\mathfrak{a}\right\|^{2}\right)},
$$

where $\mathfrak{a}$ is the cusp point $\mathcal{G}_{\mathbf{x}_{i}}(t)$ is close to. Then $t=-\log \left\|\mathbf{x}_{i}-\mathfrak{a}\right\| \in I$.

Suppose $s \in I$ is the right limit of $I$, then at $s, W\left(\mathcal{V}_{k}, t\right)$ changes from $W_{i}\left(\mathbf{x}_{i}, t\right)$ to $W_{j}\left(\mathbf{x}_{j}, t\right)$, then

$$
W_{i}\left(\mathbf{x}_{i}, t\right)=\frac{1}{h(\mathfrak{a})\left(e^{-t}+e^{t}\left\|\mathbf{x}_{i}-\mathfrak{a}\right\|^{2}\right)},
$$

is equal to

$$
W_{j}\left(\mathbf{x}_{j}, t\right)=\frac{1}{h(\mathfrak{b})\left(e^{-t}+e^{t}\left\|\mathbf{x}_{j}-\mathfrak{b}\right\|^{2}\right)} .
$$

Obviously $-\log \left\|\mathbf{x}_{i}-\mathfrak{a}\right\|<t<-\log \left\|\mathbf{x}_{j}-\mathfrak{b}\right\|$, then

$$
W_{i}\left(\mathbf{x}_{i}, t\right) \asymp \frac{1}{h(\mathfrak{a}) e^{t}\left\|\mathbf{x}_{i}-\mathfrak{a}\right\|},
$$

and

this implies that

$$
W_{j}\left(\mathbf{x}_{j}, t\right) \asymp \frac{1}{h(\mathfrak{b}) e^{-t}}
$$

$h(\mathfrak{a}) e^{t}\left\|\mathbf{x}_{i}-\mathfrak{a}\right\|^{2} \asymp e^{-t} h(\mathfrak{b}) \asymp \sqrt{h(\mathfrak{a}) h(\mathfrak{b})}\left\|\mathbf{x}_{i}-\mathfrak{a}\right\|$.

Then $W\left(\mathcal{V}_{k}, t\right)$ has a local minimum $\frac{1}{\sqrt{h(\mathfrak{b}) h(\mathfrak{a})}\left\|\mathbf{x}_{i}-\mathfrak{a}\right\|}$ when $e^{t} \asymp \frac{\sqrt{h(\mathfrak{b})}}{\sqrt{h(\mathfrak{a})}\left\|\mathbf{x}_{i}-\mathfrak{a}\right\|} . W\left(\mathcal{V}_{k}, t\right) \rightarrow \infty$ if and only if these local minima $\frac{1}{\sqrt{h(\mathfrak{b}) h(\mathfrak{a})}\left\|\mathbf{x}_{i}-\mathfrak{a}\right\|}$ tends to $\infty$ as $t \rightarrow \infty$. 
5.1. Lower bound of the Hausdorff dimension. The basic idea to get the lower bound of the Hausdorff dimension is the following: it suffices to consider the case $k=2$, because if the projection of $\mathcal{G}\left(\mathcal{V}_{k}\right)$ to the first two component is divergent, then so is $\mathcal{G}\left(\mathcal{V}_{k}\right)$. For the first component, we choose $\mathbf{x}_{1}$ such that $\operatorname{Spec}\left(\mathbf{x}_{1}\right)$ admits a subsequence $\left\{\mathfrak{a}_{i}: i \in \mathbb{N}\right\}$ such that

- $h\left(\mathfrak{a}_{i+1}\right) \asymp h^{1+\delta}\left(\mathfrak{a}_{i}\right)$ for all $i \in \mathbb{N}$, where $\delta>0$ is some constant.

- From $\mathcal{G}_{\mathbf{x}_{1}}$ leaving $\mathfrak{a}_{i}$ to it entering $\mathfrak{a}_{i+1}$, it spends at most a uniformly bounded time $C>0$.

For such $\mathbf{x}_{1}$,

$$
\left\|\mathbf{x}_{1}-\mathfrak{a}_{i}\right\| \asymp \frac{1}{\sqrt{h\left(\mathfrak{a}_{i}\right) h\left(\mathfrak{a}_{i+1}\right)}} \asymp \frac{1}{h^{1+\delta / 2}\left(\mathfrak{a}_{i}\right)},
$$

and we could divide $\mathbb{R}$ into disjoint union of a sequence of intervals $\mathbb{R}=\bigcup_{i=1}^{\infty} I_{i}$, each $I_{i}=\left[t_{i}, t_{i+1}\right)$ where

$$
e^{t_{i}} \asymp \frac{1}{\left\|\mathbf{x}_{1}-\mathfrak{a}_{i}\right\|}
$$

for each $t_{i}$, such that for $t \in I_{i}$

$$
W_{1}\left(\mathbf{x}_{1}, t\right) \asymp \frac{1}{h\left(\mathfrak{a}_{i}\right)\left(e^{-t}+e^{t}\left\|\mathbf{x}_{1}-\mathfrak{a}_{i}\right\|^{2}\right)} .
$$

For each such $\mathbf{x}_{1}$ fixed, we choose $\mathbf{x}_{2}$ on the second component inductively as follows:

- We start with a cusp $\mathfrak{b}_{p}$ for some large $p$, such that $h\left(\mathfrak{b}_{p}\right) \asymp \frac{h\left(\mathfrak{a}_{p}\right)}{\log h\left(\mathfrak{a}_{p}\right)}$, and take a neighborhood $B\left(\mathfrak{b}_{p}, \frac{1}{h\left(\mathfrak{a}_{p}\right)}\right)$. We denote $A_{p}=B\left(\mathfrak{b}_{p}, \frac{1}{h\left(\mathfrak{a}_{p}\right)}\right)$, and $\mathfrak{A}_{p}=\left\{A_{p}\right\}$.

- Suppose we have defined a collection of finitely many neighborhoods $\mathfrak{A}_{k}$, and each neighborhood of $\mathfrak{A}_{k}$ is of form $A_{k}=B\left(\mathfrak{b}_{k}, \frac{1}{h\left(\mathfrak{a}_{k}\right)}\right)$, where $\mathfrak{b}_{k}$ is some cusp point of $\Gamma_{2} \backslash \mathbb{H}^{n}$ with $h\left(\mathfrak{b}_{k}\right) \asymp \frac{h\left(\mathfrak{a}_{k}\right)}{\log h\left(\mathfrak{a}_{k}\right)}$. For each $\mathfrak{b}_{k+1} \in A_{k}$ with $h\left(\mathfrak{b}_{k+1}\right) \asymp \frac{h\left(\mathfrak{a}_{k+1}\right)}{\log h\left(\mathfrak{a}_{k+1}\right)}$, we construct the neighborhood $B\left(\mathfrak{b}_{k+1}, \frac{1}{h\left(\mathfrak{a}_{k+1}\right)}\right)$, and define $\mathfrak{A}_{k+1}$ to be the collection of all such neighborhoods. This defines $\mathfrak{A}_{n}$ inductively for all $n \in \mathbb{N}$.

Denote $\mathcal{A}_{k}=\bigcup_{A_{k} \in \mathfrak{A}_{k}} A_{k}$, and define $A_{\infty}=\bigcap_{k} \mathcal{A}_{k}$. We will prove the following lemma:

Lemma 5.1. Fix $\mathbf{x}_{1}$ and define $A_{\infty}$ as above, then for any $\mathbf{x}_{2} \in A_{\infty}, \mathcal{G}\left(\mathbf{x}_{1}, \mathbf{x}_{2}\right)$ is a divergent trajectory.

Proof. For any $\mathbf{x}_{2} \in A_{\infty}$, there exists a sequence of cusp points $\left\{\mathfrak{b}_{k}: k \geq p\right\}$ such that

- $h\left(\mathfrak{b}_{k}\right) \asymp \frac{h\left(\mathfrak{a}_{k}\right)}{\log h\left(\mathfrak{a}_{k}\right)}$, for all $k$.

- $\mathbf{x}_{2} \in B\left(\mathfrak{b}_{k}, \frac{1}{h\left(\mathfrak{a}_{k}\right)}\right)$.

Then from $e^{t} \asymp h\left(\mathfrak{a}_{k}\right) \log h\left(\mathfrak{a}_{k}\right)$ to $e^{t}=\frac{1}{\left\|\mathbf{x}_{1}-\mathfrak{a}_{k}\right\|}$,

$$
\begin{aligned}
& W_{1}\left(\mathbf{x}_{1}, t\right) \\
= & \frac{1}{h\left(\mathfrak{a}_{k}\right)\left(e^{-t}+e^{t}\left\|\mathbf{x}_{1}-\mathfrak{a}_{k}\right\|^{2}\right)} \\
\asymp & \frac{1}{h\left(\mathfrak{a}_{k}\right) e^{-t}} \\
\gg & \frac{h\left(\mathfrak{a}_{k}\right) \log h\left(\mathfrak{a}_{k}\right)}{h\left(\mathfrak{a}_{k}\right)} \\
= & \log h\left(\mathfrak{a}_{k}\right) .
\end{aligned}
$$

From $e^{t}=\frac{1}{\left\|\mathbf{x}_{1}-\mathfrak{a}_{k}\right\|}$ to $e^{t} \asymp \frac{h\left(\mathfrak{a}_{k+1}\right)}{\log h\left(\mathfrak{a}_{k+1}\right)}$,

$$
\begin{aligned}
& W_{1}\left(\mathbf{x}_{1}, t\right) \\
\asymp & \frac{1}{e^{t} h\left(\mathfrak{a}_{k}\right)\left\|\mathbf{x}_{1}-\mathfrak{a}_{k}\right\| \|^{2}} \\
\gg & \frac{\log h\left(\mathfrak{a}_{k+1}\right)}{h\left(\mathfrak{a}_{k+1} h\left(\mathfrak{a}_{k}\right)\left\|\mathbf{x}_{1}-\mathfrak{a}_{k}\right\|^{2}\right.} \\
\asymp & \log h\left(\mathfrak{a}_{k+1}\right) .
\end{aligned}
$$

From $e^{t} \asymp \frac{h\left(\mathfrak{a}_{k+1}\right)}{\log h\left(\mathfrak{a}_{k+1}\right)} \asymp h\left(\mathfrak{b}_{k+1}\right)$ to $e^{t}=\frac{1}{\left\|\mathbf{x}_{2}-\mathfrak{b}_{k+1}\right\|} \asymp h\left(\mathfrak{a}_{k+1}\right)$,

$$
W_{2}\left(\mathbf{x}_{2}, t\right) \asymp \frac{e^{t}}{h\left(\mathfrak{b}_{k+1}\right)} \asymp \frac{e^{t} \log h\left(\mathfrak{a}_{k+1}\right)}{h\left(\mathfrak{a}_{k+1}\right)},
$$


and it is increasing. We claim that at some $\tau_{k+1}$ such that

$$
e^{2 \tau_{k+1}} \asymp \frac{h\left(\mathfrak{b}_{k+1}\right)}{h\left(\mathfrak{a}_{k}\right)\left\|\mathbf{x}_{1}-\mathfrak{a}_{k}\right\|^{2}} \asymp h\left(\mathfrak{b}_{k+1}\right) h\left(\mathfrak{a}_{k+1}\right),
$$

$W\left(\mathcal{V}_{2}, t\right)$ admits a local minimum and

$$
W\left(\mathcal{V}_{2}, \tau_{k+1}\right)=W_{2}\left(\mathbf{x}_{2}, \tau_{k+1}\right) \asymp \sqrt{\frac{h\left(\mathfrak{a}_{k+1}\right)}{h\left(\mathfrak{b}_{k+1}\right)}} \asymp \sqrt{\log h\left(\mathfrak{a}_{k+1}\right)} .
$$

Since $\sqrt{\log h\left(\mathfrak{a}_{k+1}\right)} \rightarrow \infty$ as $t \rightarrow \infty$, we have the diagonal geodesic ray $\mathcal{G}\left(\mathbf{x}_{1}, \mathbf{x}_{2}\right)$ is divergent.

Therefore, once we choose $\mathbf{x}_{1}$ as above and then choose a $\mathbf{x}_{2} \in A_{\infty}$ for this fixed $\mathbf{x}_{1}$, we will get a divergent trajectory. We will then compute the Hausdorff dimension of the collection of such $\mathbf{x}_{1}$ 's, and for each $\mathbf{x}_{1}$ fixed, we will give the Hausdorff dimension of $A_{\infty}$, by the following lemma, the Hausdorff dimension of divergent trajectories is at least the sum of them:

Lemma 5.2 (Marstrand Slicing Theorem). Let $A$ and $B$ be metric spaces, and let $C$ be a subset of the direct product $A \times B$. Assume that the projection of $C$ onto $A, \operatorname{Proj}_{A}(C)$ has Hausdorff dimension at least $\alpha>0$, and for every $a \in \operatorname{Proj}_{A}(C)$, if we define

$$
B_{a}=C \cap(\{a\} \times B)
$$

then the Hausdorff dimension $\operatorname{dim}_{H}\left(B_{a}\right) \geq \beta>0$ for all $a \in \operatorname{Proj}_{A}(C)$, then we have

$$
\operatorname{dim}_{H} C \geq \alpha+\beta \text {. }
$$

Remark 5.3. The reader may see [11, Section 1.4], [12], and [8, Theorem 5.8] for the detail of this theorem.

We at first prove that for each fixed $\mathbf{x}_{1}$ as above, the set $A_{\infty}$ defined as above has Hausdorff dimension $n-1$ :

Theorem 5.4. Given constants $\delta>0$ and $C>0$, let $\mathbf{x}_{1} \in \mathbb{R}^{n-1}$ satisfy the following condition: its spectrum $\operatorname{Spec}\left(\mathbf{x}_{1}\right)$ admits a sebsequence $\left\{\mathfrak{a}_{k}: k \in \mathbb{N}\right\}$, such that

- $h\left(\mathfrak{a}_{k+1}\right) \asymp h^{1+\delta}\left(\mathfrak{a}_{k}\right)$ for all $k \in \mathbb{N}$.

- From $\mathcal{G}_{\mathbf{x}_{1}}$ leaving $\mathfrak{a}_{k}$ to it entering $\mathfrak{a}_{k+1}$, it spends at most time $C$.

According to this $\mathbf{x}_{1}$, we construct $\mathfrak{A}_{k}$ inductively as above, and then define

$$
\mathcal{A}_{k}=\bigcup_{A \in \mathfrak{A}_{k}} A
$$

and

$$
A_{\infty}=\bigcap_{k} \mathcal{A}_{k}
$$

then the Hausdorff dimension of $A_{\infty}$

$$
\operatorname{dim}_{H} A_{\infty} \geq n-1
$$

We introduce the notion of Cantor-like collection of compact subsets of $\mathbb{R}^{n-1}$ as follows:

Definition 5.5. Starting with a bounded closed subset $A_{0}$ with positive Lebesgue measure, a Cantor-like countable collection $\mathfrak{A}$ is the union of finite collections $\mathfrak{A}_{k}$ of compact subsets of $A_{0}$, for $k \in \mathbb{N}$, satisfying the following conditions:

(1) $\mathfrak{A}_{0}=\left\{A_{0}\right\}$

(2) every $\mathfrak{A}_{k}$ is a finite collection of disjoint compact subsets of $A_{0}$.

(3) for every $k \geq 1$, for every $A \in \mathfrak{A}_{k}$, we can find some $B \in \mathfrak{A}_{k-1}$ such that $A \subset B$.

(4) let $d_{k}(\mathfrak{A})=\sup _{A \in \mathfrak{A}_{k}} \operatorname{diam}(A)$, where $\operatorname{diam}(A)$ denotes the diameter of $A$, then $d_{k}(\mathfrak{A}) \rightarrow 0$ as $k \rightarrow \infty$. For a Cantor-like collection $\mathfrak{A}=\bigcup_{k=0}^{\infty} \mathfrak{A}_{k}$, let $\mathcal{A}_{k}=\bigcup_{A \in \mathfrak{A}_{k}} A$, and $A_{\infty}=\bigcap_{k=0}^{\infty} \mathcal{A}_{k}$, and we define $\Delta_{k}(\mathfrak{A})$ as folows:

$$
\Delta_{k}(\mathfrak{A})=\inf _{B \in \mathfrak{A}_{k}} \frac{m\left(B \cap \mathcal{A}_{k+1}\right)}{m(B)}
$$

where $m(\cdot)$ denotes the Lebesgue measure of $\mathbb{R}^{n-1}$. 
The basic tool of the proof is the following theorem:

Theorem 5.6. (See [11, Section 4.1]) Let $\mathfrak{A}=\bigcup_{k=0}^{\infty} \mathfrak{A}_{k}$ be a Cantor-like collection of compact subsets of $A_{0}$, and let $\mathcal{A}_{k}, A_{\infty}, d_{k}(\mathfrak{A})$ and $\Delta_{k}(\mathfrak{A})$ be as above, then we have the Hausdorff dimension of $A_{\infty}$

$$
\operatorname{dim}_{H}\left(A_{\infty}\right) \geq n-1-\limsup _{j \rightarrow \infty} \frac{\sum_{i=0}^{j-1} \log \left(\frac{1}{\Delta_{i}(\mathfrak{A})}\right)}{\log \left(\frac{1}{d_{j}(\mathfrak{A})}\right)}
$$

Remark 5.7. The statement given in [11, Section 4.1] is more general than the version above, and the result was proved in [15] and [16].

Now we are ready to prove Theorem 5.4.

Proof of Theorem 5.4. To apply Theorem [5.6, we need to estimate $\Delta_{k}(\mathfrak{A})$.

Given each $A_{k} \in \mathfrak{A}_{k}$, suppose $A_{k}=B\left(\mathfrak{b}_{k}, \frac{1}{h\left(\mathfrak{a}_{k}\right)}\right)$, where $h\left(\mathfrak{b}_{k}\right) \asymp \frac{h\left(\mathfrak{a}_{k}\right)}{\log h\left(\mathfrak{a}_{k}\right)}$. We need to count how many cusp points $\mathfrak{b}_{k+1} \in A_{k}$ with $h\left(\mathfrak{b}_{k+1}\right) \asymp \frac{h\left(\mathfrak{a}_{k+1}\right)}{\log h\left(\mathfrak{a}_{k+1}\right)}$.

To do this, we at first find a successive cusp point $\mathfrak{b}_{k}^{\prime}$ of $\mathfrak{b}_{k}$ in $A_{k}$ such that

$$
h\left(\mathfrak{b}_{k}^{\prime}\right) \asymp h\left(\mathfrak{a}_{k}\right) \log h\left(\mathfrak{a}_{k}\right),
$$

then

$$
\left\|\mathfrak{b}_{k}-\mathfrak{b}_{k}^{\prime}\right\| \asymp \frac{1}{h\left(\mathfrak{a}_{k}\right)} .
$$

Let constants $T, \Upsilon, A_{1}, A_{2}$ and $A_{3}$ be as in Theorem 4.1 and define $A_{k}^{\prime}=B\left(\mathfrak{b}_{k}^{\prime}, \frac{A_{3}}{h\left(\mathfrak{b}_{k}^{\prime}\right)}\right)$, then $A_{k}^{\prime} \subset A_{k}$, and applying Theorem 4.1 for $t>T$, there are at least $\Upsilon e^{(n-1) t}$ cusp points $\mathfrak{b}_{k+1}$ 's in $A_{k}^{\prime}$ with $h\left(\mathfrak{b}_{k+1}\right) \in$ $\left[A_{1} e^{t} h\left(\mathfrak{b}_{k}^{\prime}\right), A_{2} e^{t} h\left(\mathfrak{b}_{k}^{\prime}\right)\right]$. Let

$$
e^{t}=\frac{h\left(\mathfrak{b}_{k+1}\right)}{h\left(\mathfrak{b}_{k}^{\prime}\right)} \asymp \frac{h^{\delta}\left(\mathfrak{a}_{k}\right)}{\log ^{2} h\left(\mathfrak{a}_{k}\right)},
$$

we get the number of choices for $\mathfrak{b}_{k+1}$ is $\asymp \frac{h^{(n-1) \delta}\left(\mathfrak{a}_{k}\right)}{\log ^{2(n-1)} h\left(\mathfrak{a}_{k}\right)}$.

Therefore

so

$$
\frac{1}{\Delta_{k}(\mathfrak{A})} \asymp \frac{\log ^{2(n-1)} h\left(\mathfrak{a}_{k}\right)}{h^{(n-1) \delta}\left(\mathfrak{a}_{k}\right)} h^{(n-1) \delta}\left(\mathfrak{a}_{k}\right)=\log ^{2(n-1)} h\left(\mathfrak{a}_{k}\right),
$$

$$
\log \left(\frac{1}{\Delta_{k}(\mathfrak{A})}\right)=O(k)
$$

and because

$$
\log \left(\frac{1}{d_{k}(\mathfrak{A})}\right)=(1+\delta)^{k} \log h\left(\mathfrak{a}_{0}\right)+O(k)
$$

we have

$$
\begin{aligned}
& \operatorname{dim}_{H} A_{\infty} \\
\geq & n-1-\limsup _{j \rightarrow \infty} \frac{\sum_{i=0}^{j-1} O(i)}{(1+\delta)^{j} \log h\left(\mathfrak{a}_{0}\right)+O(j)} \\
= & n-1-\lim \sup _{j \rightarrow \infty} \frac{O\left(j^{2}\right)}{(1+\delta)^{j} \log h\left(\mathfrak{a}_{0}\right)+O(j)} \\
= & n-1 .
\end{aligned}
$$

This completes the proof.

We give the lower bound of the Hausdorff dimension of collection of eligible $\mathbf{x}_{1}$ 's in the following proposition:

Proposition 5.8. For every $\delta>0$, we define the $\mathfrak{D}_{\delta}$ to be the collection of $\mathbf{x}_{1} \in \mathbb{R}^{n-1}$ satisfying the following:

$$
\operatorname{dim}_{H} \mathfrak{D}_{\delta} \geq \frac{n-1}{2+\delta}
$$


Proof. We will at first construct the Cantor-like collection $\mathfrak{A}=\bigcup \mathfrak{A}_{k}$.

Let the constant $C>0$ be the same as those in Corollary 3.22. Starting with a cusp point $\mathfrak{a}_{0}$ with height $h\left(\mathfrak{a}_{0}\right)$ large enough, we define $A_{0}$ to be the closed ball centered at $\mathfrak{a}_{0}$ with radius $\frac{1}{h^{1+\delta / 2}\left(\mathfrak{a}_{0}\right)}$, i.e.,

$$
A_{0}=B\left(\mathfrak{a}_{0}, \frac{1}{h^{1+\delta / 2}\left(\mathfrak{a}_{0}\right)}\right)
$$

We define $\mathfrak{A}_{0}=\left\{A_{0}\right\}$.

Suppose we have defined $\mathfrak{A}_{k}$ for $k \geq 0$, and every $A_{k} \in \mathfrak{A}_{k}$ is of form $B\left(\mathfrak{a}_{k}, \frac{1}{h^{1+\delta / 2}\left(\mathfrak{a}_{k}\right)}\right)$. We fix one such $A_{k}$. We take $\mathfrak{a}_{k+1}$ to be a successive cusp point of $\mathfrak{a}_{k}$ (see Definition 3.20$)$ such that $h\left(\mathfrak{a}_{k+1}\right) \asymp h^{1+\delta}\left(\mathfrak{a}_{k}\right)$, and define $A_{k+1}=B\left(\mathfrak{a}_{k+1}, \frac{1}{h^{1+\delta / 2}\left(\mathfrak{a}_{k+1}\right)}\right)$. From Proposition 3.21, we have that

$$
\left\|\mathfrak{a}_{k+1}-\mathfrak{a}_{k}\right\| \asymp \frac{1}{\sqrt{h\left(\mathfrak{a}_{k}\right) h\left(\mathfrak{a}_{k+1}\right)}} \asymp \frac{1}{h^{1+\delta / 2}\left(\mathfrak{a}_{k}\right)},
$$

so $A_{k+1} \subset A_{k}$ if we choose the constants appropriately in the approximation

$$
h\left(\mathfrak{a}_{k+1}\right) \asymp h^{1+\delta}\left(\mathfrak{a}_{k}\right) .
$$

We take all possible $A_{k}$ 's in $\mathfrak{A}_{k}$ and construct all possible $A_{k+1}$ 's as above, and define $\mathfrak{A}_{k+1}$ to be the collection of all such $A_{k+1}$ 's.

This finishes the inductive construction of $\mathfrak{A}=\bigcup_{k=0}^{\infty} \mathfrak{A}_{k}$, and thus $\mathcal{A}_{k}=\bigcup_{A \in \mathfrak{A}_{k}} A$ and $A_{\infty}=\bigcap_{k=0}^{\infty} \mathcal{A}_{k}$ are defined accordingly.

We will prove that $A_{\infty} \subset \mathfrak{D}_{\delta}$. Take any $\mathbf{x} \in A_{\infty}$, then there exists a sequence $\left\{A_{k}=B\left(\mathfrak{a}_{k}, \frac{1}{h^{1+\delta / 2}\left(\mathfrak{a}_{k}\right)}\right) \in\right.$ $\left.\mathfrak{A}_{k}: n \in \mathbb{N}\right\}$, such that $\mathbf{x} \in \bigcap_{k=0}^{\infty} A_{k}$. By Corollary [3.22, the geodesic ray $\mathcal{G}_{\mathbf{x}}$ enters $\mathfrak{a}_{k}$ consequently, and moreover, from leaving $\mathfrak{a}_{k}$ to entering $\mathfrak{a}_{k+1}$, it spends at most time $C$. Because $h\left(\mathfrak{a}_{k+1}\right) \asymp h^{1+\delta}\left(\mathfrak{a}_{k}\right)$, this shows that $\mathbf{x} \in \mathfrak{D}_{\delta}$.

Next we will apply Theorem [5.6 to give the lower bound of the Hausdorff dimension of $A_{\infty}$.

Take any $A_{k} \in \mathfrak{A}_{k}$ of form $B\left(\mathfrak{a}_{k}, \frac{1}{h^{1+\delta / 2}\left(\mathfrak{a}_{k}\right)}\right)$, suppose that

$$
\mathfrak{a}_{k}=\gamma \xi \infty
$$

we want to count how many successive cusp points $\mathfrak{a}_{k+1}$ 's we could choose. From Definition 3.20, every $\mathfrak{a}_{k+1}$ is of form

$$
\mathfrak{a}_{k+1}=\gamma \xi u(\mathbf{n}) \xi^{-1} s \xi^{\prime} \infty
$$

and from Proposition 3.21, $h\left(\mathfrak{a}_{k+1}\right) \asymp h\left(\mathfrak{a}_{k}\right)\|\mathbf{n}\|^{2}$, therefore we have $\|\mathbf{n}\| \asymp h^{\delta / 2}\left(\mathfrak{a}_{k}\right)$. So the number of choices for $\mathfrak{a}_{k+1}$ is $\asymp h^{(n-1) \delta / 2}\left(\mathfrak{a}_{k}\right)$. Therefore

$$
\Delta_{k}(\mathfrak{A}) \asymp h^{(n-1) \delta / 2}\left(\mathfrak{a}_{k}\right) \frac{h^{(n-1)(1+\delta / 2)}\left(\mathfrak{a}_{k}\right)}{h^{(n-1)(1+\delta)(1+\delta / 2)}\left(\mathfrak{a}_{k}\right)}=h^{-(n-1)(1+\delta) \delta / 2}\left(\mathfrak{a}_{k}\right) .
$$

And

$$
d_{k}(\mathfrak{A}) \asymp \frac{1}{h^{1+\delta / 2}\left(\mathfrak{a}_{k}\right)} .
$$

So

$$
\log \left(\frac{1}{\Delta_{k}(\mathfrak{A})}\right)=(n-1)(1+\delta) \delta / 2 \log h\left(\mathfrak{a}_{k}\right)=(n-1)(1+\delta)^{k+1} \delta / 2 \log \left(h\left(\mathfrak{a}_{0}\right)\right)+O(k)
$$

and

$$
\log \left(\frac{1}{d_{k}(\mathfrak{A})}\right)=(1+\delta / 2) \log h\left(\mathfrak{a}_{k}\right)=(1+\delta / 2)(1+\delta)^{k} \log \left(h\left(\mathfrak{a}_{0}\right)\right)+O(k)
$$

where $O(k)$ denotes some quantity depending on $k$ such that $|O(k)| \ll k$ for large $k$. 
Then from Theorem 5.6 ,

$$
\begin{aligned}
& \operatorname{dim}_{H} A_{\infty} \\
\geq & n-1-\limsup _{j \rightarrow \infty} \frac{\sum_{i=0}^{j-1} \log \left(\frac{1}{\Delta_{i}(2)}\right)}{\log \left(\frac{1}{d_{j}(2)}\right)} \\
= & n-1-\limsup _{j \rightarrow \infty} \frac{\sum_{i=0}^{j-1}(1-1) \delta / 2(1+\delta)^{i+1} \log h\left(\mathfrak{a}_{0}\right)+O(i)}{(1+\delta / 2)(1+\delta)^{j} \log h\left(\mathfrak{a}_{0}\right)+O(j)} \\
= & n-1-\limsup _{j \rightarrow \infty} \frac{(n-1)(1+\delta)^{j+1} / 2 \log h\left(\mathfrak{a}_{0}\right)+O\left(j^{2}\right)}{(1+\delta / 2)(1+\delta)^{j} \log h\left(\mathfrak{a}_{0}\right)+O(j)} \\
= & n-1-\frac{(n-1)(1+\delta)}{2+\delta}=\frac{n-1}{2+\delta .}
\end{aligned}
$$

This completes the proof.

Combining Theorem 5.4, Proposition 5.8 and Lemma 5.2, we get that

$$
\operatorname{dim}_{H} \mathfrak{B}_{k} \geq(k-1)(n-1)+\frac{n-1}{2+\delta}
$$

for all $\delta>0$. By letting $\delta \rightarrow 0$, we show that

$$
\operatorname{dim}_{H} \mathfrak{B}_{k} \geq k(n-1)-\frac{n-1}{2}
$$

5.2. Upper bound of the Hausdorff dimension. The basi idea to get the upper bound of the Hausdorff dimension of $\operatorname{dim}_{H} \mathfrak{B}_{k}$ is the following:

At first we choose a small constant $\rho>0$, and define

$$
E(\rho)=\left\{\mathcal{V}_{k} \in\left(\mathbb{R}^{n-1}\right)^{k}: W\left(\mathcal{V}_{k}, t\right) \geq \rho^{-1} \quad \text { for all } t>0 \text { large enough }\right\},
$$

then obviously $\mathfrak{B}_{k} \subset E(\rho)$. We then construct an indexed self-similar covering $(\mathcal{B}, J, \varrho)$ of $E(\rho)$, which is defined as follows:

Definition 5.9. Let $\mathcal{B}$ be a countable covering of a subset $E \subset \mathbb{R}^{l}$ by bounded subsets of $\mathbb{R}^{l}$ and assume that it is indexed by some countable set $J$; let $\varrho$ be a function from the set $J$ to the set of all nonempty subsets of $J$. For any $\alpha \in J$ we write $B(\alpha)$ for the element of $\mathcal{B}$ indexed by $\alpha$. We say $(\mathcal{B}, J, \varrho)$ is an indexed self-similar covering of $E$ (the indexing function $\iota: J \rightarrow \mathcal{B}$ being implicit) if there exists a $\lambda, 0<\lambda<1$ such that for every $x \in E$ we have a sequence $\left(\alpha_{j}\right)$ of elements in $J$ satisfying

(1) $\cap B\left(\alpha_{j}\right)=\{x\}$

(2) $\operatorname{diam} B\left(\alpha_{j+1}\right)<\lambda \operatorname{diam} B\left(\alpha_{j}\right)$ for all $j$, and

(3) $\alpha_{j+1} \in \varrho\left(\alpha_{j}\right)$ for all $j$.

And then we apply the following theorem of Cheung to get the upper bound of $\operatorname{dim}_{H} E(\rho)$ :

Theorem 5.10. (See [2, Theorem 5.3]) Let $(\mathcal{B}, J, \varrho)$ be an indexed self-similar covering of a subset $E \subset \mathbb{R}^{l}$ and suppose there is an $s>0$ such that for every $\alpha \in J$

$$
\sum_{\alpha^{\prime} \in \varrho(\alpha)}\left(\operatorname{diam} B\left(\alpha^{\prime}\right)\right)^{s} \leq(\operatorname{diam} B(\alpha))^{s}
$$

Then $\operatorname{dim}_{H} E \leq s$.

So our first step is to construct the indexed self-similar covering $(\mathcal{B}, J, \varrho)$ of $E(\rho)$.

The construction of $(\mathcal{B}, J, \varrho)$ basically follows from the work of Cheung (see [2]), with some minor modification.

We need some preparation before the construction.

Lemma 5.11. For any noncompact hyperbolic space $\Gamma \backslash \mathbb{H}^{n}$ with finite volume, there exists a constant $c>0$ such that for all $X>0$ large enough, and any closed ball $B \in \mathbb{R}^{n-1}$ of radius $\frac{c}{\sqrt{X}}$, there is at least one cusp of $\Gamma \backslash \mathbb{H}^{n}$ inside $B$ with height less than or equal to $X$.

Proof. Let $\mathbf{x} \in \mathbb{R}^{n-1}$ denote the center of the ball, and consider the rough spectrum of $\mathcal{G}_{\mathbf{x}}$, say $\left\{\mathfrak{a}_{i}: i \in \mathbb{N}\right\}$. Let $\mathfrak{a}_{i}$ denote the cusp with largest height less than or equal to $X$, then we have

$$
\left\|\mathbf{x}-\mathfrak{a}_{i}\right\| \asymp \frac{1}{\sqrt{h\left(\mathfrak{a}_{i}\right) h\left(\mathfrak{a}_{i+1}\right)}} \underset{22}{\sqrt{X}}
$$


since $h\left(\mathfrak{a}_{i+1}\right) \geq X$ and $h\left(\mathfrak{a}_{i}\right) \gg 1$. Therefore there exists some constant $c>0$ such that $\mathfrak{a}_{i}$ is inside the ball centered at $\mathbf{x}$ with radius $\frac{c}{\sqrt{X}}$.

This proves the lemma.

Remark 5.12. For each component $\Gamma_{i} \backslash \mathbb{H}^{n}$ and each positive integer $N$, then the above lemma tells that there exists a countable subset $\mathfrak{E}(i, N)$ of cusps of $\Gamma_{i}$ such that for the constant $c$ and some smaller costant $c^{\prime}$ such that every closed ball $B \subset \mathbb{R}^{n-1}$ of radius $\frac{c}{\sqrt{N}}$ contains at least one element of $\mathfrak{E}(i, N)$, and in every closed ball of radius $\frac{c^{\prime}}{\sqrt{N}}$ there is at most one element of $\mathfrak{E}(i, N)$. And moreover, every element in $\mathfrak{E}(i, N)$ has height less than or equal to $N$. We fix these subsets.

Definition 5.13. Let $\mathcal{Q}_{i} \subset \mathbb{R}^{n-1}$ denote the set of cusps of $\Gamma_{i}$, and $I=\{1, \ldots, k\}$. Define $J \subset \mathcal{Q}_{1} \times \cdots \times$ $\mathcal{Q}_{k} \times I \times I$ to be the collection of elements $\left(\mathfrak{a}_{1}, \ldots, \mathfrak{a}_{k}, i, j\right)$ satisfying the following conditions:

(1) $h\left(\mathfrak{a}_{j}\right)<\rho h\left(\mathfrak{a}_{i}\right)$

(2) $h\left(\mathfrak{a}_{l}\right) \in \mathfrak{E}\left(l,\left\lfloor h\left(\mathfrak{a}_{i}\right) h\left(\mathfrak{a}_{j}\right)\right\rfloor\right)$ for other index $l$.

For all such $\left(\mathfrak{a}_{1}, \ldots, \mathfrak{a}_{k}, i, j\right)$, we denote by $B\left(\mathfrak{a}_{1}, \ldots, \mathfrak{a}_{k}, i, j\right) \subset \mathbb{R}^{n-1} \times \cdots, \times \mathbb{R}^{n-1}$ the ball centered at $\left(\mathfrak{a}_{1}, \ldots, \mathfrak{a}_{k}\right)$ with radius $\frac{c}{\sqrt{h\left(\mathfrak{a}_{i}\right) h\left(\mathfrak{a}_{j}\right)}}$ for some constant $c$, with respect to the supreme norm of the norm in $\mathbb{R}^{n-1}$, i.e.,

$$
\left\|\left(\mathbf{x}_{1}, \ldots, \mathbf{x}_{k}\right)\right\|=\max _{1 \leq i \leq k}\left\|\mathbf{x}_{i}\right\|
$$

We define $\mathcal{B}$ to be the collection of all $B\left(\mathfrak{a}_{1}, \ldots, \mathfrak{a}_{k}, i, j\right)$ for $\left(\mathfrak{a}_{1}, \ldots, \mathfrak{a}_{k}, i, j\right) \in J$. And we define

$$
A\left(\mathfrak{a}_{1}, \ldots, \mathfrak{a}_{k}, i, j\right) \subset B\left(\mathfrak{a}_{1}, \ldots, \mathfrak{a}_{k}, i, j\right)
$$

to be the neighborhood of $\left(\mathfrak{a}_{1}, \ldots, \mathfrak{a}_{k}\right)$ whose $i$ th component has radius $\frac{c}{h\left(\mathfrak{a}_{i}\right)}$ and other components have radius $\frac{c}{\sqrt{h\left(\mathfrak{a}_{i}\right) h\left(\mathfrak{a}_{j}\right)}}$.

We define $\varrho$ as follows: for $\left(\mathfrak{a}_{1}, \ldots, \mathfrak{a}_{k}, i, j\right) \in J$, we define $\varrho\left(\mathfrak{a}_{1}, \ldots, \mathfrak{a}_{k}, i, j\right) \subset J$ to be the collection of elements $\left(\mathfrak{a}_{1}^{\prime}, \ldots, \mathfrak{a}_{k}^{\prime}, j, j^{\prime}\right) \in J$ satisfying the following conditions:

(1) $\mathfrak{a}_{j}^{\prime}$ is a successive cusp point of $\mathfrak{a}_{j}$, we denote this condition by $\mathfrak{a}_{j} \mapsto \mathfrak{a}_{j}^{\prime}$.

(2) if $j^{\prime}=i$, then $h\left(\mathfrak{a}_{i}^{\prime}\right)>h\left(\mathfrak{a}_{i}\right)$ and $\left\|\mathfrak{a}_{i}^{\prime}-\mathfrak{a}_{i}\right\| \leq \frac{c}{h\left(\mathfrak{a}_{i}\right)}$

(3) $h\left(\mathfrak{a}_{i}\right)<h\left(\mathfrak{a}_{j}^{\prime}\right)$ and $h\left(\mathfrak{a}_{j}\right)<h\left(\mathfrak{a}_{j^{\prime}}^{\prime}\right)$.

(4) $A\left(\mathfrak{a}_{1}, \ldots, \mathfrak{a}_{k}, i, j\right) \cap A\left(\mathfrak{a}_{1}^{\prime}, \ldots, \mathfrak{a}_{k}^{\prime}, j, j^{\prime}\right) \neq \emptyset$

(5) $h^{2}\left(\mathfrak{a}_{i}\right) \leq h\left(\mathfrak{a}_{j}^{\prime}\right) h\left(\mathfrak{a}_{j^{\prime}}^{\prime}\right)$.

We will prove that the above construction gives an indexed self-similar covering of $E(\sigma)$ :

Proposition 5.14. Definition 5.13 gives an indexed self-similar covering of $E(\sigma)$.

Proof. For any $\mathcal{V}_{k}=\left(\mathbf{x}_{1}, \ldots, \mathbf{x}_{2}\right) \in E(\rho)$, then we have the local minima of $W\left(\mathcal{V}_{k}, t\right)$ is always greater than $\rho^{-1}$ for $t$ large enough. Then there exists a sequence of times $\left\{t_{p}: p \in \mathbb{N}\right\}$ such that at each $t=t_{p}$ it admits a local minimum. Then from the previous argument we have at this moment, $W\left(\mathcal{V}_{k}, t\right)$ changes from $W_{i(p)}\left(\mathbf{x}_{i(p)}, t\right)$ to $W_{i(p+1)}\left(\mathbf{x}_{i(p+1)}, t\right)$ for some indices $i(p), i(p+1) \in I$. And in this case if we denote by $\mathfrak{a}(p)_{i(p)}$ and $\mathfrak{a}(p+1)_{i(p+1)}$ the corresponding cusps of $\mathcal{G}_{\mathbf{x}_{i(p)}}$ and $\mathcal{G}_{\mathbf{x}_{i(p+1)}}$ respectively, at this moment, and denote by $\mathfrak{b}(p)_{i(p)}$ and $\mathfrak{b}(p+1)_{i(p+1)}$ the next cusps of $\mathcal{G}_{\mathbf{x}_{i(p)}}$ and $\mathcal{G}_{\mathbf{x}_{i(p+1)}}$ respectively, then the previous argument tells that

$$
W\left(\mathcal{V}_{k}, t_{p}\right)=\frac{1}{\sqrt{h\left(\mathfrak{a}(p)_{i(p)}\right) h\left(\mathfrak{a}(p+1)_{i(p+1)}\right)}\left\|\mathbf{x}_{i(p)}-\mathfrak{a}(p)_{i(p)}\right\|},
$$

and

$$
e^{t_{p}} \asymp \frac{\sqrt{h\left(\mathfrak{a}(p+1)_{i(p+1)}\right)}}{\sqrt{h\left(\mathfrak{a}(p)_{i(p)}\right)}\left\|\mathbf{x}_{i(p)}-\mathfrak{a}(p)_{i(p)}\right\|} .
$$

Note that if we denote by $\mathfrak{b}(p)_{i(p)}$ the next cusp $\mathcal{G}_{\mathbf{x}_{i(p)}}$ roughly enters after leaving $\mathfrak{a}(p)_{i(p)}$, we have that $($ see Proposition 3.23

$$
\left\|\mathbf{x}_{i(p)}-\mathfrak{a}(p)_{i(p)}\right\| \asymp \frac{1}{\sqrt{23}}
$$


Then $W\left(\mathcal{V}_{k}, t_{p}\right)>\rho^{-1}$ implies that $h\left(\mathfrak{a}(p+1)_{i(p+1)}\right)<\rho^{2} h\left(\mathfrak{b}(p)_{i(p)}\right)$. Then we have a sequence of triplets

$$
\left\{\left(\mathfrak{a}(p)_{i(p)}, \mathfrak{b}(p)_{i(p)}, i(p)\right): p \in \mathbb{N}\right\}
$$

It is easy to see that $h\left(\mathfrak{a}(p)_{i(p)}\right)$ and $h\left(\mathfrak{b}(p)_{i(p)}\right)$ are both increasing with respect to $p$.

Next we define a subsequence $\left\{\left(\mathfrak{a}\left(p_{l}\right)_{i\left(p_{l}\right)}, \mathfrak{b}\left(p_{l}\right)_{i\left(p_{l}\right)}, i\left(p_{l}\right)\right): l \in \mathbb{N}\right\}$ of $\left\{\left(\mathfrak{a}(p)_{i(p)}, \mathfrak{b}(p)_{i(p)}, i(p)\right)\right\}$ as follows: we start with some large $p_{0} \in \mathbb{N}$ and suppose $p_{l}$ is defined, we define $p_{l+1}$ to be the smallest subindex $p$ such that

$$
h^{2}\left(\mathfrak{b}\left(p_{l}\right)_{i\left(p_{l}\right)}\right) \leq h\left(\mathfrak{b}(p)_{i(p)}\right) h\left(\mathfrak{a}(p+1)_{i(p+1)}\right)
$$

This defines the subsequence $\left\{\left(\mathfrak{a}\left(p_{l}\right)_{i\left(p_{l}\right)}, \mathfrak{b}\left(p_{l}\right)_{i\left(p_{l}\right)}, i\left(p_{l}\right)\right): l \in \mathbb{N}\right\}$.

From the definition it is easy to see that

$$
h^{2}\left(\mathfrak{b}\left(p_{l}\right)_{i\left(p_{l}\right)}\right) \leq h\left(\mathfrak{b}\left(p_{l+1}\right)_{i\left(p_{l+1}\right)}\right) h\left(\mathfrak{a}\left(p_{l+2}\right)_{i\left(p_{l+2}\right)}\right)
$$

for all $l \in \mathbb{N}$.

Moreover, from the definition we have

$$
h^{2}\left(\mathfrak{b}\left(p_{l}\right)_{i\left(p_{l}\right)}\right)>h\left(\mathfrak{b}\left(p_{l+1}-1\right)_{i\left(p_{l+1}-1\right)}\right) h\left(\mathfrak{a}\left(p_{l+1}\right)_{i\left(p_{l+1}\right)}\right)>\frac{1}{\rho^{2}} h^{2}\left(\mathfrak{a}\left(p_{l+1}\right)_{i\left(p_{l+1}\right)}\right)
$$

this implies that

$$
h\left(\mathfrak{a}\left(p_{l+1}\right)_{i\left(p_{l+1}\right)}\right)<\rho h\left(\mathfrak{b}\left(p_{l}\right)_{i\left(p_{l}\right)}\right)
$$

For simplicity, in the following argument, we write $l$ for $p_{l}$. Then we have

$$
h^{2}\left(\mathfrak{b}(l)_{i(l)}\right) \leq h\left(\mathfrak{b}(l+1)_{i(l+1)}\right) h\left(\mathfrak{a}(l+2)_{i(l+2)}\right)
$$

and

Then we consider

$$
h\left(\mathfrak{a}(l+1)_{i(l+1)}\right)<\rho h\left(\mathfrak{b}(l)_{i(l)}\right)
$$

for each $l \in \mathbb{N}$ such that

$$
\mathfrak{u}(l)=\left(\mathfrak{c}(l)_{1}, \ldots, \mathfrak{c}(l)_{k}, i(l), i(l+1)\right)
$$

(1) $\mathfrak{c}(l)_{i(l)}=\mathfrak{b}(l)_{i(l)}$

(2) $\mathfrak{c}(l)_{i(l+1)}=\mathfrak{a}(l+1)_{i(l+1)}$

(3) for other index $j$, we choose $\mathfrak{c}(l)_{j}$ to be a cusp in $\mathfrak{E}\left(j,\left\lfloor h\left(\mathfrak{c}(l)_{i(l)}\right) h\left(\mathfrak{c}(l)_{i(l+1)}\right)\right\rfloor\right)$ such that $\left\|\mathbf{x}_{j}-\mathfrak{c}(l)_{j}\right\| \leq$ $\frac{c}{\sqrt{h\left(\mathfrak{c}(l)_{i(l)}\right) h\left(\mathfrak{c}(l)_{i(l+1)}\right)}}$, this can always be done because of the property of $\mathfrak{E}(i, N)$ (see the remark after Lemma 5.11).

It can be seen from the above argument that $\mathfrak{u}(l) \in J$. Moreover, we claim that

$$
\mathcal{V}_{k} \in B\left(\mathfrak{c}(l)_{1}, \ldots, \mathfrak{c}(l)_{k}, i(l), i(l+1)\right) .
$$

At first, $\mathfrak{a}(l+1)_{i(l+1)}$ and $\mathfrak{b}(l+1)_{i(l+1)}$ are two consecutive cusps in the rough spectrum of $\mathbf{x}_{i(l+1)}$, so we have

$$
\left\|\mathbf{x}_{i(l+1)}-\mathfrak{a}(l+1)_{i(l+1)}\right\| \asymp \frac{1}{\sqrt{h\left(\mathfrak{a}(l+1)_{i(l+1)}\right) h\left(\mathfrak{b}(l+1)_{i(l+1)}\right)}} \leq \frac{1}{\sqrt{h\left(\mathfrak{a}(l+1)_{i(l+1)}\right) h\left(\mathfrak{b}(l)_{i(l)}\right)}},
$$

and

$$
\left\|\mathbf{x}_{i(l)}-\mathfrak{b}(l)_{i(l)}\right\| \ll \frac{1}{h\left(\mathfrak{b}(l)_{i(l)}\right)} \leq \frac{1}{\sqrt{h\left(\mathfrak{a}(l+1)_{i(l+1)}\right) h\left(\mathfrak{b}(l)_{i(l)}\right)}} .
$$

For any other index $j$, we have

$$
\left\|\mathbf{x}_{j}-\mathfrak{c}(l)_{j}\right\| \leq \frac{c}{\sqrt{h\left(\mathfrak{c}(l)_{i(l)}\right) h\left(\mathfrak{c}(l)_{i(l+1)}\right)}},
$$

from our choice of $\mathfrak{c}(l)_{j}$. Therefore, we may choose appropriate constant $c>0$ in the definition of $B\left(\mathfrak{c}(l)_{1}, \ldots, \mathfrak{c}(l)_{k}, i(l), i(l+1)\right)$ such that

$$
\mathcal{V}_{k} \in B\left(\mathfrak{c}(l)_{1}, \ldots, \mathfrak{c}(l)_{k}, i(l), i(l+1)\right),
$$

for all $l \in \mathbb{N}$. Moreover, from the argument above we have

$$
\mathcal{V}_{k} \in A\left(\mathfrak{c}(l)_{1}, \ldots, \mathfrak{c}(l)_{k}, i(l), i(l+1)\right) .
$$


The diameter of $B(\mathfrak{u}(l))$

Therefore

$$
\operatorname{diam} B(\mathfrak{u}(l))=\frac{2 c}{\sqrt{h\left(\mathfrak{a}(l+1)_{i(l+1)}\right) h\left(\mathfrak{b}(l)_{i(l)}\right)}}
$$

$$
\begin{aligned}
& \frac{\operatorname{diam} B(\mathfrak{u}(l+1))}{\operatorname{diam} B(\mathfrak{u}(l))} \\
= & \left(\frac{h\left(\mathfrak{a}(l+1)_{i(l+1)}\right) h\left(\mathfrak{b}(l)_{i(l)}\right)}{h\left(\mathfrak{a}(l+2)_{i(l+2)}\right) h\left(\mathfrak{b}(l+1)_{i(l+1)}\right)}\right)^{1 / 2} \\
\leq & \left(\frac{h\left(\mathfrak{a}(l+1)_{i(l+1)}\right) h\left(\mathfrak{b}(l)_{i(l)}\right)}{h^{2}\left(\mathfrak{b}(l)_{i(l)}\right)}\right)^{1 / 2} \\
\leq & \rho^{1 / 2} .
\end{aligned}
$$

This shows that $\operatorname{diam} B(\mathfrak{u}(l+1)) \leq \lambda \operatorname{diam} B(\mathfrak{u}(l))$ for $\lambda=\rho^{1 / 2}<1$. And moreover this implies that $\operatorname{diam} B(\mathfrak{u}(l)) \rightarrow 0$ as $l \rightarrow \infty$. Combining this with $\mathcal{V}_{k} \in B(\mathfrak{u}(l))$ for any $l \in \mathbb{N}$, we have that

$$
\bigcap_{l=0}^{\infty} B(\mathfrak{u}(l))=\left\{\mathcal{V}_{k}\right\}
$$

To show that $(\mathcal{B}, J, \varrho)$ is a self-similar covering of $E(\rho)$, the last thing is to verify that

$$
\left(\mathfrak{c}(l+1)_{1}, \ldots, \mathfrak{c}(l+1)_{k}, i(l+1), i(l+2)\right) \in \varrho\left(\mathfrak{c}(l)_{1}, \ldots, \mathfrak{c}(l)_{k}, i(l), i(l+1)\right) .
$$

At first, $\mathfrak{a}(l+1)_{i(l+1)} \mapsto \mathfrak{b}(l+1)_{i(l+1)}$ since they are consecutive cusps in the rough spectrum of $\mathbf{x}_{i(l+1)}$ (see Proposition 3.23), so the first condition is verified.

If $i(l+2)=i(l)$, then $\mathfrak{c}(l)_{i(l)}$ and $\mathfrak{c}(l+1)_{i(l)}$ are both in the rough spectrum of $\mathbf{x}_{i(l)}$, this ensures the second condition.

The third condition is true since $h\left(\mathfrak{a}(l)_{i(l)}\right)$ and $h\left(\mathfrak{b}(l)_{i(l)}\right)$ are both increasing with respect to $l$. The fourth condition is true since $\mathcal{V}_{k} \in A(\mathfrak{u}(l)) \cap A(\mathfrak{u}(l+1))$ for all $l \in \mathbb{N}$. The last condition is directly from the definition of $\left(\mathfrak{a}(l)_{i(l)}, \mathfrak{b}(l)_{i(l)}, i(l)\right)$. Thus we prove that

$$
\left(\mathfrak{c}(l+1)_{1}, \ldots, \mathfrak{c}(l+1)_{k}, i(l+1), i(l+2)\right) \in \varrho\left(\mathfrak{c}(l)_{1}, \ldots, \mathfrak{c}(l)_{k}, i(l), i(l+1)\right) .
$$

This shows that $(\mathcal{B}, J, \varrho)$ is a self-similar covering of $E(\rho)$.

Now we are ready to apply Theorem 5.10 to give the upper bound of the Hausdorff dimension of $E(\rho)$.

We will need the following lemma concerning the upper bound of the number of cusp points inside a ball $B \subset \mathbb{R}^{n-1}$ with height bounded by $X>0$ :

Lemma 5.15. For a noncompact hyperbolic space $\Gamma \backslash \mathbb{H}^{n}$ and a closed ball $B \subset \mathbb{R}^{n-1}$, we denote by $F_{B}(t)$ the number of cusps of $\Gamma$ inside $B$ with height less than or equal to $t$ for $t>0$. Then there exists some constant $\omega>0$ such that for every $X>0$ large enough, we have

$$
\int_{1}^{X} \frac{1}{t^{(n-1) / 2}} d F_{B}(t) \leq \omega X^{(n-1) / 2} \operatorname{Vol}(B) .
$$

Proof. We may choose $X$ large enough such that $\frac{1}{\sqrt{X}} \ll \operatorname{diam}(B)$. Note that the left hand hand of the inequality above is equal to

$$
\sum_{\mathfrak{a}} \frac{1}{h^{(n-1) / 2}(\mathfrak{a})}
$$

where $\mathfrak{a}$ runs over all cusps of $\Gamma$ inside $B$ with height less than or equal to $X$ (we may assume that every cusp has height greater or equal to 1 without loss of generality since the height is uniformly bounded from 0 ). For every two cusps $\mathfrak{a}_{1}, \mathfrak{a}_{2} \in B$, both with height less than or equal to $X$, we will have

$$
\left\|\mathfrak{a}_{1}-\mathfrak{a}_{2}\right\| \gg \frac{1}{\sqrt{h\left(\mathfrak{a}_{1}\right) h\left(\mathfrak{a}_{2}\right)}} \geq \frac{1}{\sqrt{X h\left(\mathfrak{a}_{i}\right)}},
$$

for $i=1,2$. Therefore there exists some constant $c$ such that if we denote by $B(\mathfrak{a})$ a ball centered at $\mathfrak{a}$ with radius $\frac{c}{\sqrt{X h(\mathfrak{a})}}$, for every cusp $\mathfrak{a}$ inside $B$ with height less than or equal to $X$, then we have every ball is disjoint from others. And since we choose $X$ large enough, we have

$$
\bigcup_{\mathfrak{a}} B(\mathfrak{a}) \subset B^{\prime}
$$


where $B^{\prime}$ is some larger ball sharing the center of $B$ and has diameter $\tilde{c} \operatorname{diam}(B)$ for some other constant $\tilde{c}$. This implies that

$$
\sum_{\mathfrak{a}} \operatorname{Vol}(B(\mathfrak{a})) \leq \operatorname{Vol}\left(B^{\prime}\right)=\tilde{c}^{n-1} \operatorname{Vol}(B)
$$

This is equivalent to

$$
\sum_{\mathfrak{a}} \frac{c^{n-1}}{X^{(n-1) / 2} h^{(n-1) / 2}(\mathfrak{a})} \leq \zeta \operatorname{Vol}(B)
$$

for some constant $\zeta>0$. The above inequality implies that

$$
\sum_{\mathfrak{a}} \frac{1}{h^{(n-1) / 2}(\mathfrak{a})} \leq \omega X^{(n-1) / 2} \operatorname{Vol}(B),
$$

and this implies the conclusion immediately.

Now let us apply Theorem 5.10 to give the upper bound of the Hausdorff dimension of $E(\rho)$ :

Theorem 5.16. For $\rho>0$ small enough, we have

$$
\operatorname{dim}_{H} E(\rho) \leq(k-1)(n-1)+(n-1) / 2+\rho^{1 / 8} .
$$

Proof. For a fixed $\left(\mathfrak{a}_{1}, \ldots, \mathfrak{a}_{k}, i, j\right) \in J$, and $s=k(n-1)-(n-1) / 2+\rho^{1 / 8}$, we want to estimate

$$
\sum_{\left(\mathfrak{a}_{1}^{\prime}, \ldots, \mathfrak{a}_{k}^{\prime}, j, j^{\prime}\right) \in \varrho\left(\mathfrak{a}_{1}, \ldots, \mathfrak{a}_{k}, i, j\right)}\left(\frac{\operatorname{diam} B\left(\mathfrak{a}_{1}^{\prime}, \ldots, \mathfrak{a}_{k}^{\prime}, j, j^{\prime}\right)}{\operatorname{diam} B\left(\mathfrak{a}_{1}, \ldots, \mathfrak{a}_{k}, i, j\right)}\right)^{s} .
$$

Let us denote

$$
\varrho_{l}\left(\mathfrak{a}_{1}, \ldots, \mathfrak{a}_{k}, i, j\right)=\left\{\left(\mathfrak{a}_{1}^{\prime}, \ldots, \mathfrak{a}_{k}^{\prime}, j, l\right) \in \varrho\left(\mathfrak{a}_{1}, \ldots, \mathfrak{a}_{k}, i, j\right)\right\} .
$$

Then the above summation can be separated as

$$
\sum_{l} \sum_{\varrho_{l}\left(\mathfrak{a}_{1}, \ldots, \mathfrak{a}_{k}, i, j\right)}\left(\frac{h\left(\mathfrak{a}_{i}\right) h\left(\mathfrak{a}_{j}\right)}{h\left(\mathfrak{a}_{j}^{\prime}\right) h\left(\mathfrak{a}_{l}^{\prime}\right)}\right)^{s / 2}
$$

Let us put $\frac{h\left(\mathfrak{a}_{j}^{\prime}\right)}{h\left(\mathfrak{a}_{j}\right)}=a$ and $\frac{h\left(\mathfrak{a}_{\mathfrak{l}}^{\prime}\right)}{h\left(\mathfrak{a}_{i}\right)}=b$, then we have $a>\rho^{-1}$ and $b<\rho a$.

We separate the choice of $l$ into two case:

(1) $l=i$ : let us denote by $H(a)$ the number of cusps $\mathfrak{a}_{j}^{\prime}$ such that $\mathfrak{a}_{j} \mapsto \mathfrak{a}_{j}$ and $h\left(\mathfrak{a}_{j}^{\prime}\right) \leq h\left(\mathfrak{a}_{j}\right)$, then it is easily seen that $H(a) \asymp a^{(n-1) / 2}$ (suppose $\mathfrak{a}_{j}=\gamma \xi \infty$, then $\mathfrak{a}_{j}^{\prime}=\gamma \xi u(\mathbf{n}) \xi^{-1} s \xi^{\prime} \infty$, choice of $s$ and $\xi^{\prime}$ are both finite, and $\|\mathbf{n}\|^{2} \leq a$ implies the number of choices for $\mathbf{n}$ is $\left.\asymp a^{(n-1) / 2}\right)$. And since

$$
\left\|\mathfrak{a}_{i}^{\prime}-\mathfrak{a}_{i}\right\| \leq \frac{c}{h\left(\mathfrak{a}_{i}\right)}
$$

We denote by $F(b)$ the number of cusps in side the ball $B\left(\mathfrak{a}_{i}, \frac{c}{h\left(\mathfrak{a}_{i}\right)}\right)$ with height less than or equal to $b h\left(\mathfrak{a}_{i}\right)$. And once we fix $a$ and $b$, then for any other component $w$, cusps are elements in $\mathfrak{E}\left(w,\left\lfloor h\left(\mathfrak{a}_{j}^{\prime}\right) h\left(\mathfrak{a}_{i}^{\prime}\right)\right\rfloor\right)$ inside the ball centered at $\mathfrak{a}_{w}$ with radius $\frac{c}{\sqrt{h\left(\mathfrak{a}_{i}\right) h\left(\mathfrak{a}_{j}\right)}}$, from the definition of $\mathfrak{E}(w, N)$, we have the number of choices for $\mathfrak{a}_{w}^{\prime}$ is

$$
\asymp\left(\frac{h\left(\mathfrak{a}_{i}^{\prime}\right) h\left(\mathfrak{a}_{j}^{\prime}\right)}{h\left(\mathfrak{a}_{i}\right) h\left(\mathfrak{a}_{j}\right)}\right)^{(n-1) / 2}=(a b)^{(n-1) / 2} .
$$

Therefore the summation

$$
\sum_{\varrho_{i}\left(\mathfrak{a}_{1}, \ldots, \mathfrak{a}_{k}, i, j\right)}\left(\frac{h\left(\mathfrak{a}_{i}\right) h\left(\mathfrak{a}_{j}\right)}{h\left(\mathfrak{a}_{i}^{\prime}\right) h\left(\mathfrak{a}_{j}^{\prime}\right)}\right)^{s / 2}
$$

can be estimated as the following summation

$$
\int_{a>\rho^{-1}} \int_{1}^{\rho a}(a b)^{(k-2)(n-1) / 2} \frac{1}{(a b)^{s / 2}} d F(b) d H(a)
$$


By simplifying it we have

$$
\int_{a>\rho^{-1}} a^{(k-2)(n-1) / 2-s / 2} \int_{1}^{\rho a} b^{(k-1)(n-1) / 2-s / 2} \frac{1}{b^{(n-1) / 2}} d F(b) d H(a) .
$$

Let $G(x)=\int_{1}^{x} \frac{1}{b^{(n-1) / 2}} d F(b)$, then from Lemma 5.15, we have

$$
G(x) \ll x^{(n-1) / 2} .
$$

This is true since in $B\left(\mathfrak{a}_{i}, c\right), \mathfrak{a}_{i}$ is the cusp with smallest height.

Then the above integral equals

$$
\begin{aligned}
& \int_{a>\rho^{-1}} \int_{1}^{\rho a} a^{(k-2)(n-1) / 2-s / 2} b^{(k-1)(n-1) / 2-s / 2} d G(b) d H(a) \\
= & \int_{a>\rho^{-1}} a^{(k-2)(n-1) / 2-s / 2}\left((\rho a)^{(k-1)(n-1) / 2-s / 2} G(\rho a)-\int_{1}^{\rho a} G(b) d b^{(k-1)(n-1) / 2-s / 2}\right) d H(a) \\
= & \int_{a>\rho^{-1}} a^{(k-2)(n-1) / 2-s / 2}\left((\rho a)^{(k-1)(n-1) / 2-s / 2} G(\rho a)\right. \\
& \left.+(s / 2-(k-1)(n-1) / 2) \int_{1}^{\rho a} b^{(k-1)(n-1) / 2-s / 2-1} G(b) d b\right) d H(a) \\
\ll & \int_{a>\rho^{-1}} a^{(k-2)(n-1) / 2-s / 2}\left[(\rho a)^{(k-1)(n-1) / 2-s / 2}(\rho a)^{(n-1) / 2}\right. \\
& \left.+(s / 2-(k-1)(n-1) / 2) \int_{1}^{\rho a} b^{(k-1)(n-1) / 2-s / 2-1} b^{(n-1) / 2} d b\right] d H(a) \\
= & \int_{a>\rho^{-1}} a^{(k-2)(n-1) / 2-s / 2}\left((\rho a)^{k(n-1) / 2-s / 2}\right. \\
& \left.+\frac{s / 2-(k-1)(n-1) / 2}{k(n-1) 2-s / 2}(\rho a)^{k(n-1) / 2-s / 2}\right) \mathrm{d} H(a) \\
= & \frac{\rho^{k(n-1) / 2-s / 2}(n-1)}{k(n-1)-s} \int_{a>\rho^{-1}} a^{(k-1)(n-1)-s} \mathrm{~d} H(a) \\
= & \frac{\rho^{k(n-1) / 2-s / 2}(n-1)}{k(n-1)-s}\left[\left(a^{(k-1)(n-1)-s} H(a)\right)_{\rho^{-1}}^{\infty}-\int_{a>\rho^{-1}} H(a) \mathrm{d} a^{(k-1)(n-1)-s}\right] .
\end{aligned}
$$

Since $H(a) \asymp a^{(n-1) / 2}$, the above integral asymptotically equals:

$$
\begin{aligned}
& \frac{\rho^{k(n-1) / 2-s / 2}(n-1)}{k(n-1)-s}\left[\left(a^{(k-1)(n-1)-s} a^{(n-1) / 2}\right)_{\rho^{-1}}^{\infty}\right. \\
& \left.+(s-(k-1)(n-1)) \int_{a>\rho^{-1}} a^{(k-1)(n-1)-s-1+(n-1) / 2} \mathrm{~d} a\right] \\
= & \frac{\rho^{k(n-1) / 2-s / 2}(n-1)}{k(n-1)-s}\left(-(\rho)^{s-(k-1)(n-1)-(n-1) / 2}\right. \\
& \left.+\frac{s-(k-1)(n-1)}{s-(k-1)(n-1)-(n-1) / 2}(\rho)^{s-(k-1)(n-1)-(n-1) / 2}\right) \\
= & \frac{(n-1)^{2}}{2[s-(n-1)(k-1)-(n-1) / 2][k(n-1)-s]} \rho^{k(n-1) / 2-s / 2} \rho^{s-(k-1)(n-1)-(n-1) / 2} .
\end{aligned}
$$

(2) $l \neq i$ : let $H(a)$ be as above, and let $F(b)$ denote the number of cusps $\mathfrak{a}_{l}^{\prime}$ such that

$$
\left\|\mathfrak{a}_{l}^{\prime}-\mathfrak{a}_{l}\right\| \leq \frac{c}{\sqrt{h\left(\mathfrak{a}_{i}\right) h\left(\mathfrak{a}_{j}\right)}},
$$

and $h\left(\mathfrak{a}_{l}^{\prime}\right) \leq b h\left(\mathfrak{a}_{i}\right)$ Then from Lemma 5.15 tells that

$$
\int_{b \leq X} \frac{1}{\left(b h\left(\mathfrak{a}_{i}\right)\right)^{(n-1) / 2}} \mathrm{~d} F(b) \ll\left(X h\left(\mathfrak{a}_{i}\right)\right)^{(n-1) / 2} \frac{1}{\left(h\left(\mathfrak{a}_{i}\right) h\left(\mathfrak{a}_{j}\right)\right)^{(n-1) / 2}},
$$

which implies

$$
\int_{b \leq X} \frac{1}{b^{(n-1) / 2}} d F(b) \ll X^{(n-1) / 2}\left(\frac{h\left(\mathfrak{a}_{i}\right)}{h\left(\mathfrak{a}_{j}\right)}\right)^{(n-1) / 2} .
$$

We denote $G(X)=\int_{b \leq X} \frac{1}{b^{(n-1) / 2}} d F(b)$, then we have

$$
G(X) \ll X^{(n-1) / 2}\left(\frac{h\left(\mathfrak{a}_{i}\right)}{h\left(\mathfrak{a}_{j}\right)}\right)^{(n-1) / 2} .
$$

Now we fix $a$ and $b$ as above, for subindex $w \neq j, i, l, \mathfrak{a}_{w}^{\prime} \in \mathfrak{E}\left(w,\left\lfloor h\left(\mathfrak{a}_{j}^{\prime}\right) h\left(\mathfrak{a}_{l}^{\prime}\right)\right\rfloor\right)$ and

$$
\left\|\mathfrak{a}_{w}^{\prime}-\mathfrak{a}_{w}\right\| \leq \frac{c}{\sqrt{h\left(\mathfrak{a}_{i}\right) h\left(\mathfrak{a}_{j}\right)}}
$$

the number of choices for $\mathfrak{a}_{w}^{\prime}$ is asymptotically equal to

$$
\left(\frac{h\left(\mathfrak{a}_{j}^{\prime}\right) h\left(\mathfrak{a}_{l}^{\prime}\right)}{h\left(\mathfrak{a}_{i}\right) h\left(\mathfrak{a}_{j}\right)}\right)^{(n-1) / 2}=(a b)^{(n-1) / 2}
$$


For index $i$ since

$$
\left\|\mathfrak{a}_{i}^{\prime}-\mathfrak{a}_{i}\right\| \leq \frac{c}{h\left(\mathfrak{a}_{i}\right)}
$$

the number of choices for $\mathfrak{a}_{i}^{\prime}$ is asymptotically equal to

$$
\left(\frac{h\left(\mathfrak{a}_{j}^{\prime}\right) h\left(\mathfrak{a}_{l}^{\prime}\right)}{h^{2}\left(\mathfrak{a}_{i}\right)}\right)^{(n-1) / 2}=(a b)^{(n-1) / 2}\left(\frac{h\left(\mathfrak{a}_{j}\right)}{h\left(\mathfrak{a}_{i}\right)}\right)^{(n-1) / 2} .
$$

Thus the summation

$$
\sum_{\varrho_{l}\left(\mathfrak{a}_{1}, \ldots, \mathfrak{a}_{k}, i, j\right)}\left(\frac{h\left(\mathfrak{a}_{i}\right) h\left(\mathfrak{a}_{j}\right)}{h\left(\mathfrak{a}_{j}^{\prime}\right) h\left(\mathfrak{a}_{l}^{\prime}\right)}\right)^{s / 2}
$$

can be estimated as

$$
\begin{aligned}
& \int_{a>\rho^{-1}} \int_{0}^{\rho a}\left(\frac{h\left(\mathfrak{a}_{j}\right)}{h\left(\mathfrak{a}_{i}\right)}\right)^{(n-1) / 2}(a b)^{(k-2)(n-1) / 2-s / 2} \mathrm{~d} F(b) \mathrm{d} H(a) \\
= & \left(\frac{h\left(\mathfrak{a}_{j}\right)}{h\left(\mathfrak{a}_{i}\right)}\right)^{(n-1) / 2} \int_{a>\rho^{-1}} a^{(k-2)(n-1) / 2-s / 2} \int_{0}^{\rho a} b^{(k-2)(n-1) / 2-s / 2} \mathrm{~d} F(b) \mathrm{d} H(a) \\
= & \left(\frac{h\left(\mathfrak{a}_{j}\right)}{h\left(\mathfrak{a}_{i}\right)}\right)^{(n-1) / 2} \int_{a>\rho^{-1}} a^{(k-2)(n-1) / 2-s / 2} \int_{0}^{\rho a} b^{(k-1)(n-1) / 2-s / 2} \mathrm{~d} G(b) \mathrm{d} H(a) \\
= & \left(\frac{h\left(\mathfrak{a}_{j}\right)}{h\left(\mathfrak{a}_{i}\right)}\right)^{(n-1) / 2} \int_{a>\rho^{-1}} a^{(k-2)(n-1) / 2-s / 2}\left[(\rho a)^{(k-1)(n-1) / 2-s / 2} G(\rho a)\right. \\
& \left.+(s / 2-(k-1)(n-1) / 2) \int_{0}^{\rho a} G(b) b^{(k-1)(n-1) / 2-s / 2-1} \mathrm{~d} b\right] \mathrm{d} H(a) \\
\ll & \left(\frac{h\left(\mathfrak{a}_{j}\right)}{h\left(\mathfrak{a}_{i}\right)}\right)^{(n-1) / 2} \int_{a>\rho^{-1}} a^{(k-2)(n-1) / 2-s / 2}\left[\left(\frac{h\left(\mathfrak{a}_{i}\right)}{h\left(\mathfrak{a}_{j}\right)}\right)^{(n-1) / 2}(\rho a)^{k(n-1) / 2-s / 2}\right. \\
& \left.+\left(\frac{h\left(\mathfrak{a}_{i}\right)}{h\left(\mathfrak{a}_{j}\right)}\right)^{(n-1) / 2}(s / 2-(k-1)(n-1) / 2) \int_{0}^{\rho a} b^{k(n-1) / 2-s / 2-1} \mathrm{~d} b\right] \mathrm{d} H(a) \\
= & \frac{\rho^{k(n-1) / 2-s / 2}(n-1)}{k(n-1)-s} \int_{a>\rho^{-1}} a^{(k-1)(n-1)-s} \mathrm{~d} H(a) \\
= & \frac{\rho^{k(n-1) / 2-s / 2}(n-1)}{k(n-1)-s}\left[\left(a^{(k-1)(n-1)-s} H(a)\right)_{\rho^{-1}}^{\infty}\right. \\
& \left.+(s-(k-1)(n-1)) \int_{a>\rho^{-1}} H(a) a^{(k-1)(n-1)-s-1} \mathrm{~d} a\right] \\
\asymp & \frac{(n-1)^{2}}{2[s-(n-1)(k-1)-(n-1) / 2][k(n-1)-s]} \rho^{k(n-1) / 2-s / 2} \rho^{s-(k-1)(n-1)-(n-1) / 2} .
\end{aligned}
$$

We omit several steps in the last estimate since it is the same as the first case.

Summing up the two cases above, we have the summation

$$
\begin{aligned}
& \sum_{\left(\mathfrak{a}_{1}^{\prime}, \ldots, \mathfrak{a}_{k}^{\prime}, j, j^{\prime}\right) \in \varrho\left(\mathfrak{a}_{1}, \ldots, \mathfrak{a}_{k}, i, j\right)}\left(\frac{\operatorname{diam} B\left(\mathfrak{a}_{1}^{\prime}, \ldots, \mathfrak{a}_{k}^{\prime}, j, j^{\prime}\right)}{\operatorname{diam} B\left(\mathfrak{a}_{1}, \ldots, \mathfrak{a}_{k}, i, j\right)}\right)^{s} \\
& \asymp \frac{(n-1)^{2}}{2[s-(n-1)(k-1)-(n-1) / 2][k(n-1)-s]} \rho^{k(n-1) / 2-s / 2} \rho^{s-(k-1)(n-1)-(n-1) / 2} \text {. }
\end{aligned}
$$

For any $\rho>0$ small, and $s=(k-1)(n-1)+(n-1) / 2+\rho^{1 / 8}$, we have:

$$
\begin{aligned}
& \frac{(n-1)^{2}}{2[s-(n-1)(k-1)-(n-1) / 2][k(n-1)-s]} \rho^{k(n-1) / 2-s / 2} \rho^{s-(k-1)(n-1)-(n-1) / 2} \\
\asymp & \rho^{(n-1) / 4+\rho^{1 / 8} / 2-1 / 8} \\
\leq & \rho^{1 / 8} .
\end{aligned}
$$

Thus for $\rho>0$ small enough, we prove that

$$
\sum_{\left(\mathfrak{a}_{1}^{\prime}, \ldots, \mathfrak{a}_{k}^{\prime}, j, j^{\prime}\right) \in \varrho\left(\mathfrak{a}_{1}, \ldots, \mathfrak{a}_{k}, i, j\right)}\left(\frac{\operatorname{diam} B\left(\mathfrak{a}_{1}^{\prime}, \ldots, \mathfrak{a}_{k}^{\prime}, j, j^{\prime}\right)}{\operatorname{diam} B\left(\mathfrak{a}_{1}, \ldots, \mathfrak{a}_{k}, i, j\right)}\right)^{s} \leq 1,
$$

for $s=(k-1)(n-1)+(n-1) / 2+\rho^{1 / 8}$. From Theorem 5.10, we show that

$$
\operatorname{dim}_{H} E(\rho) \leq(k-1)(n-1)+(n-1) / 2+\rho^{1 / 8} .
$$


The above theorem shows that

$$
\operatorname{dim}_{H} \mathfrak{B}_{k} \leq(k-1)(n-1)+(n-1) / 2+\rho^{1 / 8},
$$

for all $\rho>0$ small enough. By letting $\rho \rightarrow 0$, we have:

$$
\operatorname{dim}_{H} \mathfrak{B}_{k} \leq(k-1)(n-1)+(n-1) / 2 .
$$

Proof of Theorem 1.1, Combining (5.1) and (5.2), we complete the proof of Proposition 2.2, This concludes Theorem 1.1 from the reduction argument in Section 2.

\section{REFERENCES}

[1] Yitwah Cheung. Hausdorff dimension of the set of nonergodic directions. Annals of mathematics, pages 661-678, 2003.

[2] Yitwah Cheung. Hausdorff dimension of the set of points on divergent trajectories of a homogeneous flow on a product space. Ergodic Theory and Dynamical Systems, 27(01):65-85, 2007.

[3] Yitwah Cheung. Hausdorff dimension of the set of singular pairs. Annals of mathematics, 173(01):127-167, 2011.

[4] YITWAH CHEUNG and NICOLAS CHEVALLIER. Hausdorff dimension of singular vectors. preprint, 2014.

[5] Yitwah Cheung, Pascal Hubert, and Howard Masur. Dichotomy for the hausdorff dimension of the set of nonergodic directions. Inventiones mathematicae, 183(2):337-383, 2011.

[6] SG Dani. Divergent trajectories of flows on homogeneous spaces and diophantine approximation. J. reine angew. Math, 359(55-89):102, 1985.

[7] Manfred Einsiedler, Shirali Kadyrov, and Anke Pohl. Escape of mass and entropy for diagonal flows in real rank one situations. arXiv preprint arXiv:1110.0910, 2011.

[8] Kenneth J Falconer. The geometry of fractal sets, volume 85. Cambridge university press, 1986.

[9] H Garland and MS Raghunathan. Fundamental domains for lattices in rank one semisimple lie groups. Proceedings of the National Academy of Sciences, 62(2):309-313, 1969.

[10] Alexander Gorodnik and Nimish A Shah. Khinchin's theorem for approximation by integral points on quadratic varieties. Mathematische Annalen, 350(2):357-380, 2011.

[11] DY Kleinbock and GA Margulis. Bounded orbits of nonquasiunipotent flows on homogeneous spaces. American Mathematical Society Translations, pages 141-172, 1996.

[12] JM Marstrand. The dimension of cartesian product sets. In Mathematical Proceedings of the Cambridge Philosophical Society, volume 50, pages 198-202. Cambridge Univ Press, 1954.

[13] Howard Masur. Hausdorff dimension of the set of nonergodic foliations of a quadratic differential. Duke Math. J, 66(3):387$442,1992$.

[14] Howard Masur and John Smillie. Hausdorff dimension of sets of nonergodic measured foliations. Annals of Mathematics, pages 455-543, 1991.

[15] Curt McMullen. Area and hausdorff dimension of julia sets of entire functions. Transactions of the American Mathematical Society, 300(1):329-342, 1987.

[16] Mariusz Urbanski. The hausdorff dimension of the set of points with nondense orbit under a hyperbolic dynamical system. Nonlinearity, 4(2):385, 1991.

Mathematical Sciences Research Institute, Berkeley, CA, 94720, U.S.A.

Current address: Einstein Institute of Mathematics, Hebrew University of Jerusalem, Jerusalem, 9190401, Israel

E-mail address: yang.lei@mail.huji.ac.il 